\title{
Review Article \\ Electrical Switching in Thin Film Structures Based on Transition Metal Oxides
}

\author{
A. Pergament, G. Stefanovich, V. Malinenko, and A. Velichko \\ Faculty of Physical Engineering, Petrozavodsk State University, Petrozavodsk 185910, Russia \\ Correspondence should be addressed to A. Pergament; aperg@psu.karelia.ru and G. Stefanovich; gstef@yandex.ru
}

Received 6 April 2015; Revised 19 July 2015; Accepted 18 August 2015

Academic Editor: Ram N. P. Choudhary

Copyright (C) 2015 A. Pergament et al. This is an open access article distributed under the Creative Commons Attribution License, which permits unrestricted use, distribution, and reproduction in any medium, provided the original work is properly cited.

Electrical switching, manifesting itself in the nonlinear current-voltage characteristics with S- and N-type NDR (negative differential resistance), is inherent in a variety of materials, in particular, transition metal oxides. Although this phenomenon has been known for a long time, recent suggestions to use oxide-based switching elements as neuristor synapses and relaxationoscillation circuit components have resumed the interest in this area. In the present review, we describe the experimental facts and theoretical models, mainly on the basis of the Mott transition in vanadium dioxide as a model object, of the switching effect with special emphasis on the emerging applied potentialities for oxide electronics.

\section{Introduction}

The phenomenon of electrical switching [1] is a rapid and reversible transition between a high-resistance (HR) state and a low-resistance (LR) state affected by an applied electric field. Two main types of the switching effect are described by an $\mathrm{N}$-shaped or an S-shaped $I-V$ characteristic and denoted as N-NDR and S-NDR [2], respectively, where the abbreviation "NDR" means "negative differential resistance" (see Figure 1). The two stable states are also frequently referred to as an OFF state (which is equivalent to HR for the case of S-NDR and to LR for N-NDR) and, correspondingly, an ON state (Figure 2).

The switching effect with S-NDR was originally found and investigated in chalcogenide-glass semiconductors (CGS) [1, 3]. Electronic switching in amorphous CGS thin films can be divided into two general types [4]: (a) monostable threshold switching, in which continuous electrical power is required to maintain the highly conducting ON state and (b) bistable memory switching, in which both $\mathrm{ON}$ and OFF states can be maintained without electrical power. In other words, for threshold switching, the OFF-toON transition is absolutely reversible and repetitive, whereas for memory switching a large voltage pulse is usually applied in the erase operation cycle to switch a device from the $\mathrm{ON}$ back to the OFF state.

We note apropos that recently the studies of bistable switching in CGS have been renewed in connexion with the development of phase change memory (PCM) technology. At present, PCM is considered to be a promising candidate for next generation nonvolatile memory. As compared with flash memory, PCM provides larger endurance, considerably faster read and write speeds, and lower power consumption [5].

Thus, current-voltage characteristics of some CGS-based thin film sandwich structures after electrical forming (the process of electroforming, EF, will be described below) are S-shaped. Similar phenomena have also been then observed in oxides [6-8] (mainly those of transition metals) and oxide glasses, amorphous and polycrystalline $\mathrm{Si}$, and other semiconductors [2], halides, nitrides, sulphides of metals, carbon-containing materials, particularly, nanotubes, organic compounds, such as conducting polymers and self-assembled monolayers, and many other materials (see [4] and references therein).

Note that S-NDR and N-NDR switching devices, in theirs operation, are the analogues of the conventional semiconductor devices such as, for example, thyristors and tunnel diodes, respectively. However, in contrast to the latter, the CGS-based and oxide-based switching devices do not require formation of $\mathrm{p}-\mathrm{n}$ junctions.

Finally, we remind the reader that memory switching in oxide thin film structures is now considered, on a par with PCM, as a promising approach to the design of new generation of computer memory, oxide ReRAM (resistive 


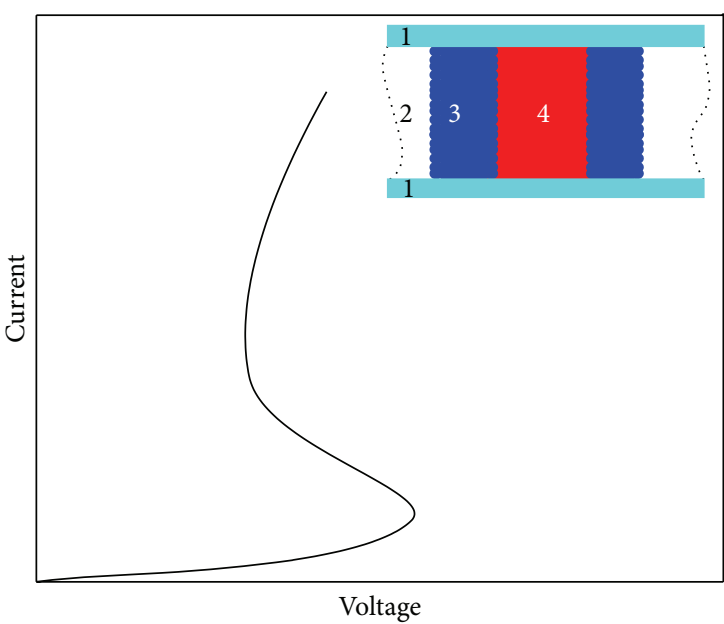

(a)

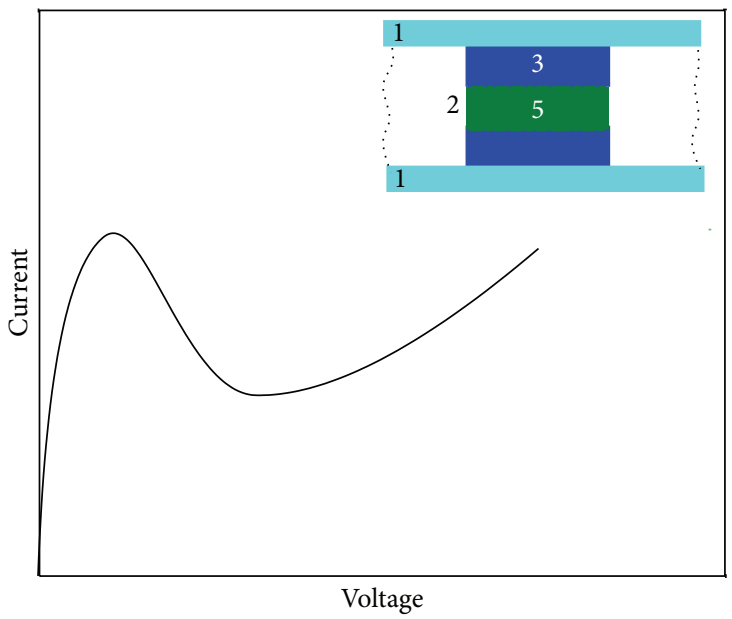

(b)

FIGURE 1: Schematic $I-V$ curves for two types of NDR which corresponds to the regions in the curves where $\mathrm{d} V / \mathrm{d} I<0$. Inserts show sketches of a high-current filament (a) and a high-field domain (b) inherent to S- and N-NDR, respectively [2] (where (1) is metal electrodes, (2) is insulating (e.g., initial oxide or amorphous CGS) film, (3) is switching channel emerging after electrical forming, (4) is current filament, and (5) is high-field domain).

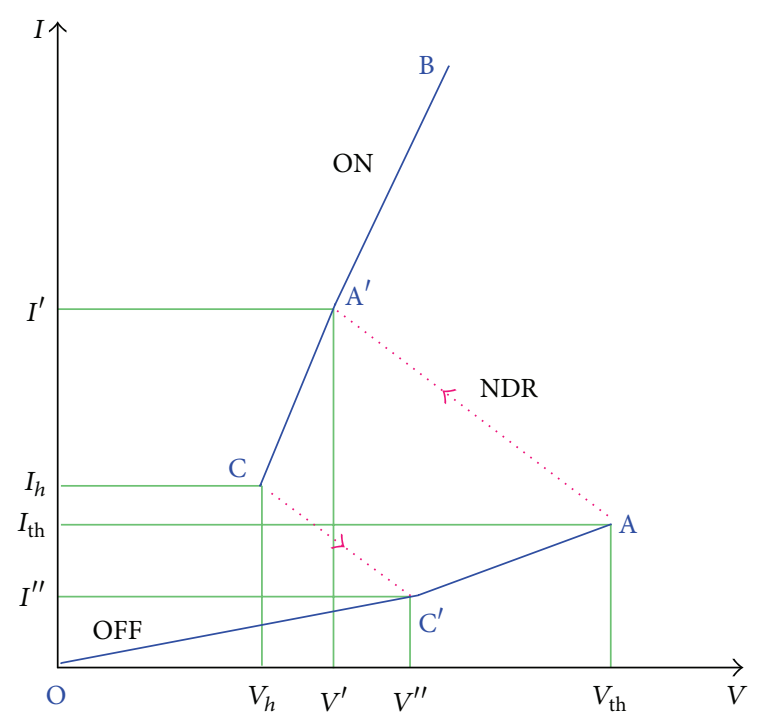

Figure 2: S-type current-voltage characteristic (O-C'-A-A'-B-A'-C$\left.\mathrm{C}^{\prime}-\mathrm{O}\right)$ of a switching device. The O- $\mathrm{C}^{\prime}-\mathrm{A}$ branch represents the OFF state, $\mathrm{C}-\mathrm{A}^{\prime}-\mathrm{B}$ the $\mathrm{ON}$ state, and $\mathrm{A}-\mathrm{A}^{\prime}$ and $\mathrm{C}-\mathrm{C}^{\prime}$ the NDR region with a hysteresis; $V_{\text {th }}$ and $V_{h}\left(I_{\text {th }}\right.$ and $\left.I_{h}\right)$ are the threshold and holding voltages (currents), respectively.

random access memory) [9]. Conceptually unsophisticated design of a cross-point metal/oxide/metal (MOM) memory cell allows easily scalable ReRAM architecture with nanometre cell dimensions. This, in turn, provides the basis for 3D integrated terabit memory with a multilayer stackable structure [10].

Among transition metal oxides (TMOs), threshold switching (without any memory effects) with an S-type $I$ $V$ characteristic has been observed in MOM structures with $\mathrm{Nb}_{2} \mathrm{O}_{5}[7,11], \mathrm{NbO}_{2}[12-14], \mathrm{TiO}_{2}[7,15], \mathrm{VO}_{2}[16], \mathrm{Ta}_{2} \mathrm{O}_{5}$
[7], Fe oxide [17], and some other TMOs [8]. As-fabricated devices rarely show threshold or memory switching effects without an initial modification of their structure, a process which is usually called EF, or just "forming" [18]. There is a striking similarity in the EF behaviour observed in a wide range of oxide, halide, sulphide, polymer, and CGS films [1821], and further we will follow the discussion of this problem in review [18].

Electroforming is achieved by the application of suitable voltage pulses which are always higher in magnitude than the subsequent operating pulses. This invariably produces an irreversible change in the characteristics of a device, often with a substantial decrease in the overall terminal resistance. The processes involved in electroforming depend on the nature and quality of the thin film, the geometrical structure of the device and, in some cases, the electrode material. The changes can be either structural, involving a movement of material from one part of the device to another, or they can be electronic, wherein a quasipermanent change in the occupancy of some electronic states takes place. The changes can occur throughout the bulk of the film or in a localized region. The most common localized effect is the formation of a fibre of highly conducting material, a switching channel. Such permanent channel formation is a consequence of temporary filamentary breakdown, often observed in metalinsulator-metal and metal-semiconductor-metal sandwich structures. However, electroforming differs from dielectric breakdown in the sense that it is a nondestructive process; that is, it rather resembles a self-healing type of breakdown [20].

The EF characteristics of different materials are generally ill defined and exhibit large variations, even for devices fabricated under the same conditions. Nevertheless, since this process is observed in a wide variety of insulating films, it is unlikely to result from a peculiarity of any one system. In 
the case of amorphous thin film structures, switching channel formation can occur via crystallization of an amorphous film, stoichiometric changes, diffusion of the electrode material into the film, or ionization of deep traps. All of these changes usually refer to a localized modification of the structure in the area of the channel. The modification of the initial structure during the forming process is the most important factor determining the subsequent switching operation. This is because, in most cases, forming creates a "new device" (within the original structure) whose characteristics determine the ensuing threshold or memory switching [18, 22].

In TMOs, a set of valence states, associated with the existence of unfilled $d$-shells in the atoms of transition metals, leads to formation of several oxide phases with different properties, ranging from metallic to insulating. On the other hand, it is specifically the behaviour of $d$-electrons in the compounds of transition metals that is responsible for the unique properties of these materials, causing strong electron-electron correlations, which play an important role in the mechanisms of such phenomena as, for example, metal-insulator transitions (MITs), high- $T_{c}$ superconductivity, colossal magnetoresistance, and multiferroicity, which are inherent to many TMOs [23-26]. That is, TMOs exhibit an astonishing array of functionalities that result from a combination of the strongly polarizable metal-oxygen bond and strong electron correlations [25].

In our works $[4,22,27-29]$, it has been shown that switching in a number of TMOs with the S-NDR exhibits several common features. Particularly, in most cases the switching effect is associated with the insulator-to-metal transition in an electric field. The channels, consisting of lower oxides exhibiting MITs, are formed in an initial oxide film during the process of EF.

In many TMOs, the switching mechanism has been shown to be associated with an electronically induced Mott MIT occurring in conditions of the nonequilibrium carrier density excess under the applied electric field. The required increase in electron density under the action of electric field might be caused by the Poole-Frenkel effect [30] or some other high-field-assisted charge carrier generation effects such as avalanche ionization and Zener breakdown, as well as Schottky, tunnel or avalanche injection from contacts, and so forth.

This review focuses on earlier obtained results, current research, the state of the art, and recent progress in the field of science and applications of the phenomenon of monostable threshold switching in oxides of transition metals. We start by discussing the switching effects in MOM structures on the basis of anodic oxide films (Sections 2.1 and 2.2) and then turn to recent results concerning electrical switching in $\mathrm{Mn}$ and Mo oxides obtained by deposition in vacuum (Section 2.3). Effects of doping to stabilize the switching parameters and unify the electroforming process are described in Section 2.4, and Section 3 is devoted to various physical mechanisms at play which could be responsible for the NDR behaviour, emphasizing the particular relationship between the switching effect and MIT, electronically induced Mott transition first of all. Further, in Section 4 we discuss applied potentialities of the TMO-based thin film devices exhibiting the
NDR phenomena, and finally, in Section 5, we will try to formulate some conclusions and outlook concerning the topic discussed. It should be stressed that the scope of the review is not to describe comprehensively all the results reported in the literature but rather to sketch out the main pertinent works and discuss the physical mechanisms involved in the phenomenon of electrical switching in transition metal oxides.

\section{Overview of Experimental Results}

2.1. Switching in Anodic Oxide Films. In this section, we present our results [4, 22, 27-31] on electroforming and switching in thin film MOM structures with oxides of transition metals (V, Ti, Fe, Nb, Mo, W, Hf, Zr, Mn, Y, and Ta) obtained by electrochemical anodic oxidation. Anodic oxidation permits the synthesis of high quality homogeneous dielectric oxide films [32] and, as a rule, anodic oxide films (AOFs) are amorphous.

The sandwich devices under study were fabricated by oxidation of the metal substrates: both polished foils and vacuum deposited layers. Au electrodes were vacuum-evaporated onto the surfaces of oxide films to complete the MOM structure (Figure 3(a)). The spring-loaded point contacts, made of gilded wire $0.5 \mathrm{~mm}$ in diameter, were also used. The oxidation was carried out electrochemically, under anodic polarization in an electrolyte: $\mathrm{Ta}, \mathrm{Nb}, \mathrm{W}, \mathrm{Zr}$, and $\mathrm{Hf}$ were anodized in $0.1 \mathrm{~N}$ aqueous solutions of phosphoric and sulphuric acids [32], while for oxidation of vanadium and molybdenum the acetone-based electrolyte was used [33]. Fe, Ti, Mn, and several of $\mathrm{Nb}$ and $\mathrm{Ta}$ samples were anodized in a $\mathrm{KNO}_{3}+$ $\mathrm{NaNO}_{3}$ eutectic melt [34] at 570-620 K. Anodic oxidation of yttrium was carried out as described in [31]. The oxide film thicknesses were typically from 50 to $200 \mathrm{~nm}$.

The current-voltage characteristics of the initial structures are nonlinear and slightly asymmetric. The resistance at zero bias, measured with the point contact, is in the range $10^{7}-10^{8} \Omega$ for the vanadium anodic oxide, which is the most highly conductive among all the materials studied. When the amplitude of the applied voltage reaches the forming voltage $V_{f}$, a sharp and irreversible increase in conductivity is observed and the $I(V)$ curve becomes S-shaped. With increasing current, the $I-V$ characteristic may change until the parameters of the switching structure are finally stabilized. The process outlined above is qualitatively similar to EF of the switching devices based on amorphous semiconductors [18]. The forming voltage depends on the temperature $\left(V_{f}\right.$ increases upon cooling) and film thickness. The thicker the film, the higher the required $V_{f}$. The value of $V_{f}$ correlates with the anodizing voltage $V_{a}$ (because $d$ is proportional to $V_{a}$ ). A similar correlation is characteristic of the AOF breakdown: the breakdown voltage is also of the order of $V_{a}$ [32].

Thus, the first stage of the forming does not differ from the conventional electrical breakdown of oxide films. However, if the postbreakdown current is limited, this results in formation of a switching channel rather than a breakdown channel. The latter would be expected to have metalliclike conductivity and no negative resistance in the $I-V$ characteristic. It is quite evident that the phase composition of this switching channel must differ from the material of the 


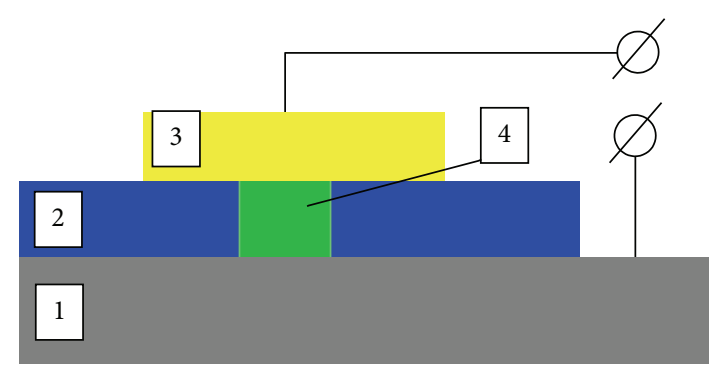

(a)

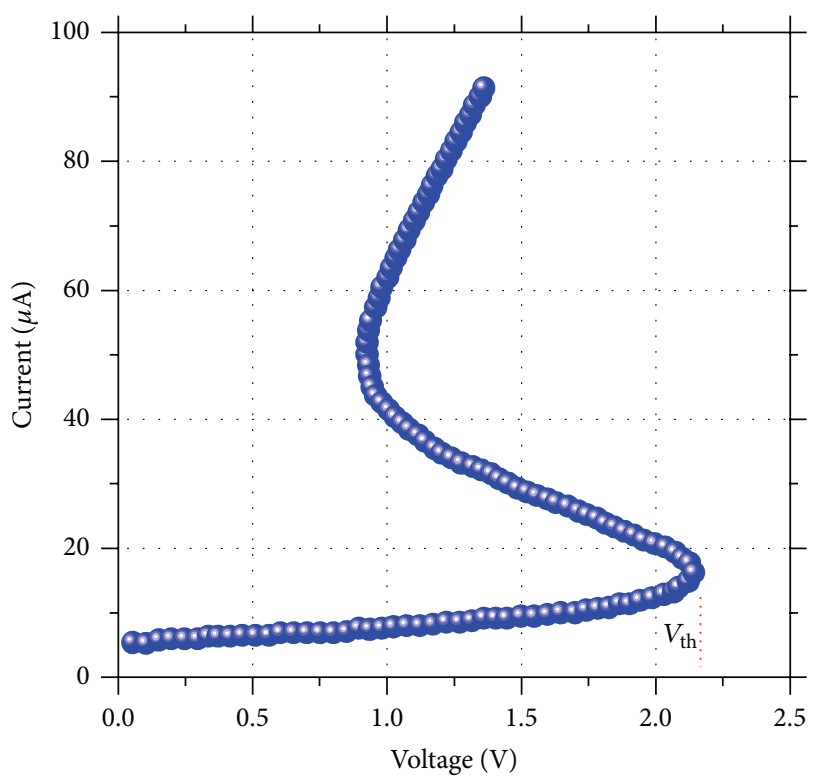

(b)

FIGURE 3: (a) The sandwich switching structure (schematic): (1) vanadium metal substrate, (2) anodic oxide film, (3) Au electrical contact, and (4) switching channel $\left(\mathrm{VO}_{2}\right.$, as will be shown below in Section 2.2); (b) the $I-V$ characteristic after EF for one of the samples at room temperature.

initial oxide film, because the channel conductivity exceeds that of an unformed structure by several orders of magnitude.

Forming does not always result in an S-type characteristic. In some instances, a transition of the structure to a high conductivity state $(R \sim 100 \Omega)$ with ohmic behaviour takes place; that is, in this case, breakdown rather than forming occurs. Breakdown is more probable in $\mathrm{Ta}_{2} \mathrm{O}_{5}, \mathrm{WO}_{3}, \mathrm{HfO}_{2}$, and $\mathrm{MoO}_{3}$ films, while in the $\mathrm{V}$ - and $\mathrm{Nb}$-based structures, it is the electroforming that results in the S-shaped $I-V$ curves in most cases. The anodic films on $\mathrm{Ti}$ and Fe hold an intermediate position. S-type switching has not been observed in $\mathrm{Zr}$, $\mathrm{Y}$, and $\mathrm{Mn}$ oxide films. EF of W-, Fe-, and Hf-based structures was carried out at $77 \mathrm{~K}$, because at room temperature breakdown took place and no switching effects were observed. The characteristic feature of switching in Ta, $\mathrm{Mo}, \mathrm{Hf}$, and, to a lesser extent, $\mathrm{W}$ oxide films is the instability of parameters and the gradual disappearance of the S-type NDR under the influence of current flow and thermal cycling.

The voltage-current characteristic for electroformed vanadium-AOF-metal structure is shown in Figure 3(b). The parameters of the structures (threshold voltage $V_{\text {th }}$ and current $I_{\text {th }}$, resistances of OFF and ON states) may vary by up to an order of magnitude from point to point for the same specimen. Such a wide range of variation of the $V_{\text {th }}$ and $R_{\text {off }}$ values, as well as the absence of correlation between these switching parameters and the parameters of the sandwich structures (the electrode material and area and the film thickness), leads to the conclusion that resistance and threshold parameters are mainly determined by the forming process. Conditions of the forming process cannot be unified in principle, because the first stage of forming is linked to breakdown, which is statistical in nature. As a result, the diameter and phase composition of the channel (and, consequently, its effective specific conductivity) vary with position in the sample. This accounts for the scatter in the parameters and for the seeming absence of their thickness dependence. The $I-V$ curves for Ti-, Nb-, and $\mathrm{W}$ based structures are also shown in Figure 4.

Similar behaviour has been observed for the other materials. On average, for $d \sim 100 \mathrm{~nm}$, a typical value of $V_{\text {th }}$ is 1 to $10 \mathrm{~V}$; that is, the threshold field is $10^{5}-10^{6} \mathrm{~V} / \mathrm{cm}$ at room temperature. The greatest differences between these oxides are observed when the temperature dependence of their threshold parameters, such as $V_{\text {th }}$, is measured. Figure 5 shows $V_{\text {th }}(T)$ curves for the sandwich structures based on iron, tungsten, vanadium, titanium, and niobium. For $\mathrm{Ta}_{2} \mathrm{O}_{5}$, $\mathrm{HfO}_{2}$, and $\mathrm{MoO}_{3}$, no reproducible results were obtained because of instabilities of their voltage-current characteristics with temperature. As the temperature increased, $V_{\text {th }}$ decreased, tending to zero at some finite temperature $T_{o}$. This is best exhibited by the vanadium and titanium oxide structures. The values of $T_{o}$ vary considerably for different oxides, but they are nearly identical for different structures of the same oxide [28]. For the $\mathrm{Nb}-\mathrm{Nb}_{2} \mathrm{O}_{5}$-metal structures, it was impossible to measure the $V_{\text {th }}(T)$ relationship over the whole temperature range, because heating above $300^{\circ} \mathrm{C}$ induced processes related to diffusion and change in the channel phase composition, leading to degradation of the switching structure. Nevertheless, it is evident from Figure 5 that, for the niobium oxide, $T_{o}$ exceeds $600 \mathrm{~K}$.

As was mentioned above, S-type switching has not been observed in $\mathrm{Zr}$, Y, and Mn oxide films. Instead, these materials have sometimes demonstrated N-NDR at $T=293 \mathrm{~K}$ ( $\mathrm{Zr}$ and 


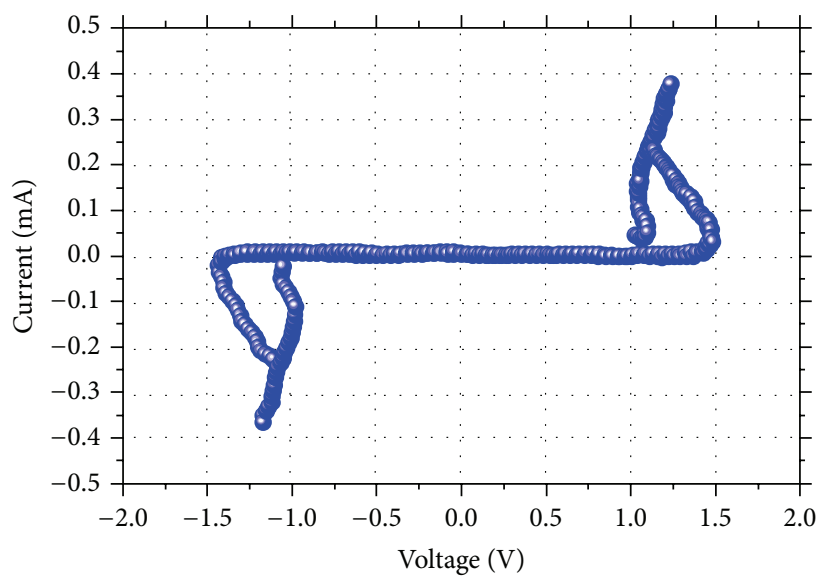

(a)

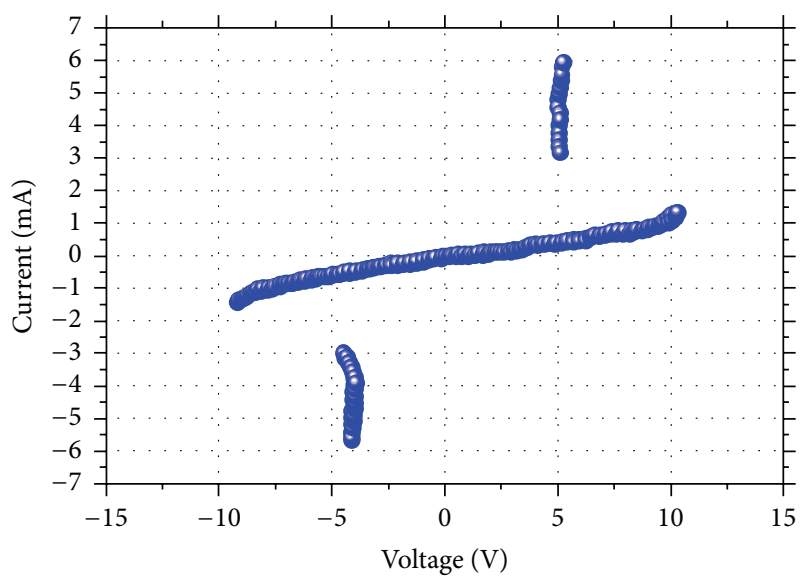

(b)

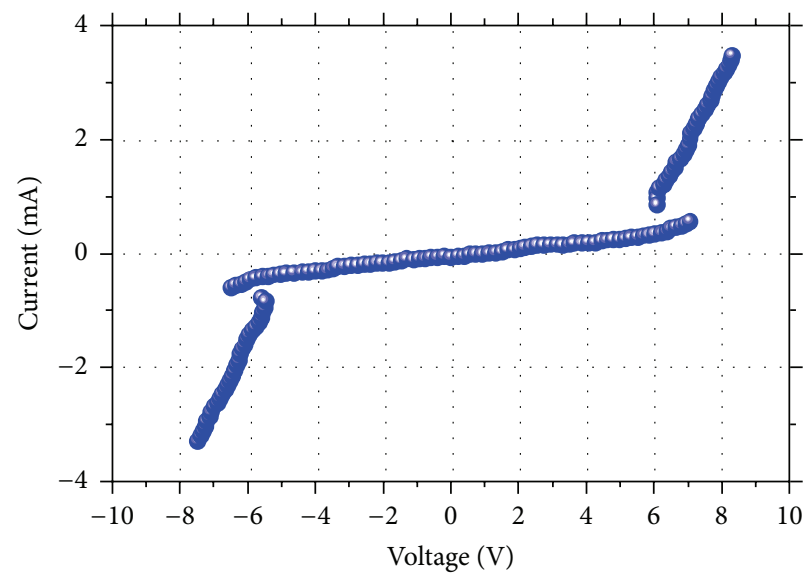

(c)

Figure 4: Current-voltage characteristics of MOM structures with AOFs on (a) Ti, (b) Nb, and (c) W [29].

Y, see Figure 6) or at $T=77 \mathrm{~K}$ (Mn oxide). The process of electroforming for these materials was hindered, like that for Ta, Hf, and Mo. Switching in Mo and Mn oxides will also be additionally discussed in Section 2.3 below.

\subsection{Switching as an Electrically Driven Metal-Insulator Transi-} tion. Next we discuss the process of electroforming in more detail. The initial AOF represents, as a rule, a highest oxide of the metal (e.g., $\mathrm{Nb}_{2} \mathrm{O}_{5}$ in the case of niobium). However, lower oxides always exist near the AOF-metal interface in the form of transition layers or as separate inclusions. This can be explained in terms of thermodynamics. Consider the following reaction:

$$
\mathrm{Nb}+2 \mathrm{Nb}_{2} \mathrm{O}_{5} \longrightarrow 5 \mathrm{NbO}_{2}
$$

The change of the Gibbs (isobaric) potential for this reaction is

$$
\Delta G_{r}=\nu_{2} \Delta G_{2}-\nu_{1} \Delta G_{1},
$$

where $\Delta G_{1}$ and $\Delta G_{2}$ are the standard isobaric potentials of formation and $v_{1}$ and $v_{2}$ are the numbers of moles of oxides which take part in the reaction. For Reaction (1), the subscripts 1 and 2 in (2) are related to $\mathrm{Nb}_{2} \mathrm{O}_{5}$ and $\mathrm{NbO}_{2}$, respectively, and $\Delta G_{r}=-163 \mathrm{~kJ} / \mathrm{mol}$ [28]. Since $\Delta G_{r}<0$, Reaction (1) proceeds spontaneously, and hence, $\mathrm{NbO}_{2}$ is always present at the $\mathrm{Nb}-\mathrm{Nb}_{2} \mathrm{O}_{5}$ interface.

Transition metals exhibit multiple oxidation states and form a number of oxides. Therefore, it is obvious that the lower oxide to be formed is mainly that with the minimum $\Delta G_{r}$. Figure 7 demonstrates the variation of $\Delta G_{r}(x)$ for $\mathrm{Fe}_{2} \mathrm{O}_{3}, \mathrm{Nb}_{2} \mathrm{O}_{5}, \mathrm{~V}_{2} \mathrm{O}_{5}, \mathrm{TiO}_{2}$, and some other oxides in reactions similar to (1), where $x$ is the stoichiometric index of oxygen. $\Delta G_{r}$ values were calculated using (2); the data on $\Delta G^{o}$ for the calculations were taken from [35]. The minima in these curves correspond to $\mathrm{Fe}_{3} \mathrm{O}_{4}, \mathrm{NbO}_{2}, \mathrm{VO}_{2}$, and $\mathrm{Ti}_{2} \mathrm{O}_{3}$; therefore, these oxides form as intermediate layers between the metal substrate and the corresponding oxide of highest valence. Real systems are far from being a plane parallel structure with two oxide layers; hence, the suggested approach is rather oversimplified. Nevertheless, the above considerations show that formation of those oxides is thermodynamically reasonable; whether it is kinetically possible can only be judged from experiment. For example, as was shown in [33], in AOF on vanadium the vanadium dioxide layer may be rather thick, and only a very thin film 


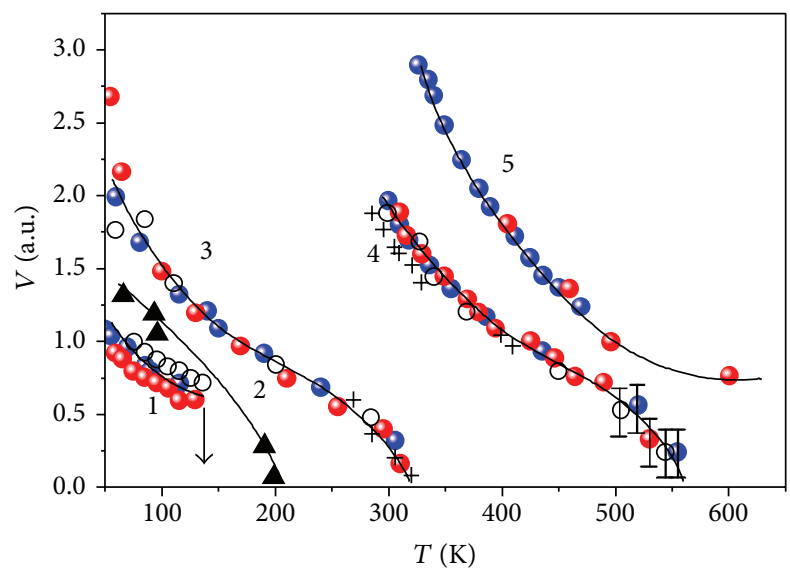

FIGURE 5: Threshold voltage in arbitrary units $V=V_{\text {th }}(T) / V_{\text {th }}\left(T^{\prime}\right)$ for the MOM structures on the basis of different oxides as a function of ambient temperature. (1) Fe, (2) W, (3) V, (4) Ti, and (5) Nb anodic oxide. Data for different samples have been averaged and normalized to a certain temperature $T^{\prime}$ which is different for different oxides $[22,27,28]$.

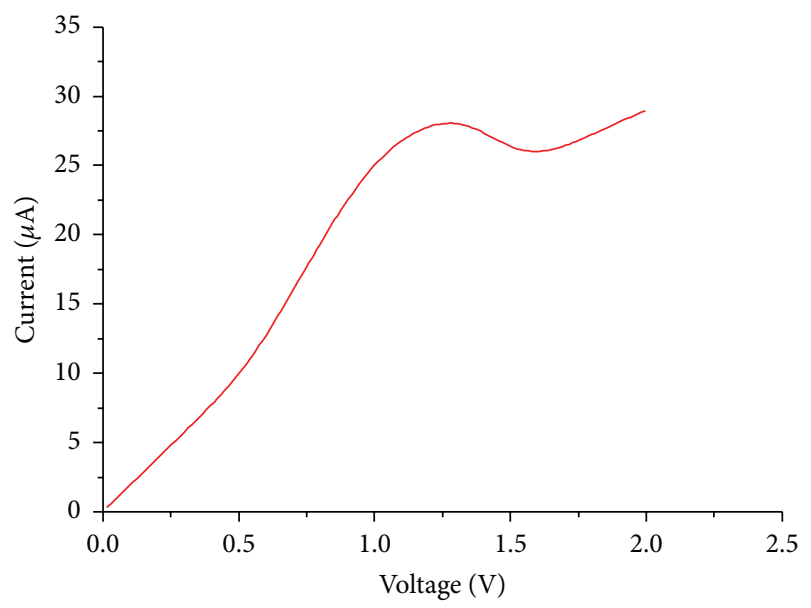

FIGURE 6: $I-V$ curve with N-NDR of the structure metal/Y anodic oxide/metal [31].

near the outer boundary is close to the $\mathrm{V}_{2} \mathrm{O}_{5}$ stoichiometry. The similar ordering of layers evidently exists in iron oxide; particularly, it is known that, in a thermal oxide of iron, the $\mathrm{Fe}_{3} \mathrm{O}_{4}$ phase usually predominates [28]. On the other hand, the AOFs on $\mathrm{Nb}$ and Ti mainly consist of $\mathrm{Nb}_{2} \mathrm{O}_{5}$ and $\mathrm{TiO}_{2}$, and $\mathrm{NbO}_{2}$ or $\mathrm{Ti}_{2} \mathrm{O}_{3}$ layers seem to be relatively thin $[27,28]$.

For the $\mathrm{W}-\mathrm{WO}_{3}$ system, a wide minimum of $\Delta G_{r}(x)$ lies in the range $x=2.7-3.0$ (Figure 7); the same behaviour seems to be characteristic of the $\mathrm{Mo}-\mathrm{MoO}_{3}$ system. The calculation for manganese oxides yields the minimum at $x=1.5$, that is, for $\mathrm{Mn}_{2} \mathrm{O}_{3}$. The curve $\Delta G_{r}(x)$ for uranium is also presented just as an example; note, however, that MITs have been reported for lower uranium oxide $\mathrm{U}_{4} \mathrm{O}_{9}$ [36]. For $\mathrm{Zr}$, Hf, Ta, and $\mathrm{Y}$ such calculations have not been made, because there exist no clear data on the thermodynamic properties of the lower oxides of those metals.

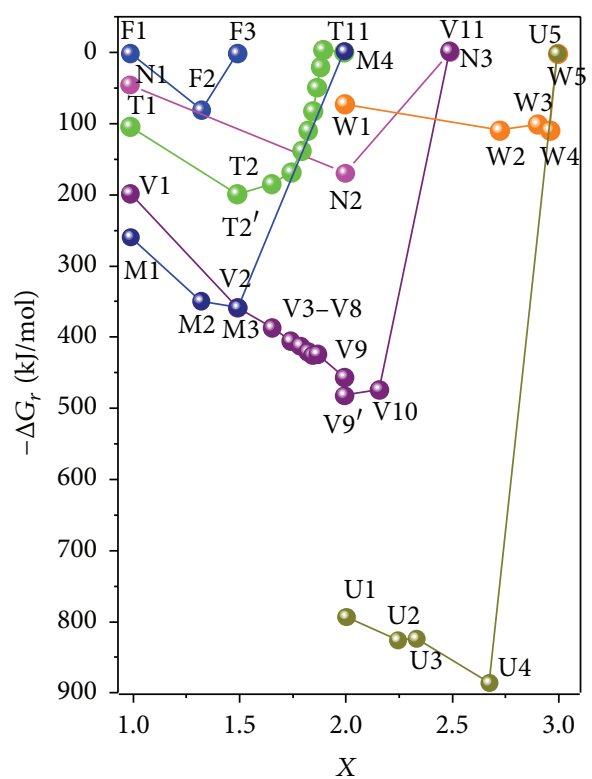

Figure 7: Gibbs potential change $\Delta G_{r}$ (per $1 \mathrm{~g}$ atom of metal) for the reaction of the highest oxide with the metal substrate versus $x$, the oxygen stoichiometric index of the lower oxide, which is formed in this reaction. Symbol indications are as follows. (i) Iron: F1-FeO; $\mathrm{F} 2-\mathrm{Fe}_{3} \mathrm{O}_{4} ; \mathrm{F} 3-\mathrm{Fe}_{2} \mathrm{O}_{3}$. (ii) Niobium: N1-NbO; N2- $\mathrm{NbO}_{2}$; $\mathrm{N} 3-\mathrm{Nb}_{2} \mathrm{O}_{5}$. (iii) Titanium: T1-TiO; T2- $\mathrm{Ti}_{2} \mathrm{O}_{3}$; T3-T10- $\mathrm{Ti}_{n} \mathrm{O}_{2 n-1}$ $(n=3-10)$; $\mathrm{T}_{11}-\mathrm{TiO}_{2}$. (iv) Vanadium: V1-VO; $\mathrm{V} 2-\mathrm{V}_{2} \mathrm{O}_{3}$; V3-V8$\mathrm{V}_{n} \mathrm{O}_{2 n-1}(n=3-8) ; \mathrm{V} 9-\mathrm{VO}_{2} ; \mathrm{V} 10-\mathrm{V}_{6} \mathrm{O}_{13} ; \mathrm{V} 11-\mathrm{V}_{2} \mathrm{O}_{5}$. (v) Tungsten: $\mathrm{W} 1-\mathrm{WO}_{2} ; \mathrm{W} 2-\mathrm{W}_{18} \mathrm{O}_{49} ; \mathrm{W} 3-\mathrm{W}_{10} \mathrm{O}_{29} ; \mathrm{W} 4-\mathrm{W}_{50} \mathrm{O}_{148} ; \mathrm{W}^{2}-\mathrm{WO}_{3}$. (vi) Manganese: $\mathrm{M} 1-\mathrm{MnO} ; \mathrm{M} 2-\mathrm{Mn}_{3} \mathrm{O}_{4} ; \mathrm{M} 3-\mathrm{Mn}_{2} \mathrm{O}_{3} ; \mathrm{M} 4-\mathrm{MnO}_{2}$. (vii) Uranium: $\mathrm{U} 1-\mathrm{UO}_{2} ; \mathrm{U} 2-\mathrm{U}_{4} \mathrm{O}_{9} ; \mathrm{U} 3-\mathrm{U}_{3} \mathrm{O}_{7} ; \mathrm{U} 4-\mathrm{U}_{3} \mathrm{O}_{8} ; \mathrm{U} 5-\mathrm{UO}_{3}$. The points $\mathrm{T} 2^{\prime}$ and $\mathrm{V} 9^{\prime}$ characterize a scatter due to the $\Delta G^{0}$ data difference $[22,28]$.

During electroforming, current-induced heating of the film under the electrode leads to a diffusion of both metal from the substrate into the film and oxygen from the outer layer to the inner one. Also the transport of metal and oxygen ions due to the applied electric field is possible. Since during electroforming (unlike thermal or electrochemical oxidation) no external oxygen is present, no reaction can take place except the reduction of the highest oxide of AOF. The reduction results in growth of the channel, consisting of lower oxide, through the film from one electrode to another. Evidently, substances with the minimum $\Delta G_{r}$ value (see Figure 7) will be predominant in the phase composition of the channel. The current increase after forming may cause further reduction of the channel material, leading to a predominance of the phases such as $\mathrm{VO}, \mathrm{NbO}$, and TiO. Many of the lowest oxides of transition metals (mono- and suboxides) exhibit metallic properties [23]; the accumulation of these phases in the channel will therefore lead to an irreversible increase in conductivity, that is, to breakdown.

The above compounds $\left(\mathrm{VO}_{2}, \mathrm{Fe}_{3} \mathrm{O}_{4}, \mathrm{Ti}_{2} \mathrm{O}_{3}\right.$, and $\left.\mathrm{NbO}_{2}\right)$ have one feature in common, namely, a metal-insulator phase transition [24]. The MIT phenomenon is characterized by the sharp and reversible change in the conductivity at a certain temperature $T_{t}$; for instance, vanadium dioxide at 


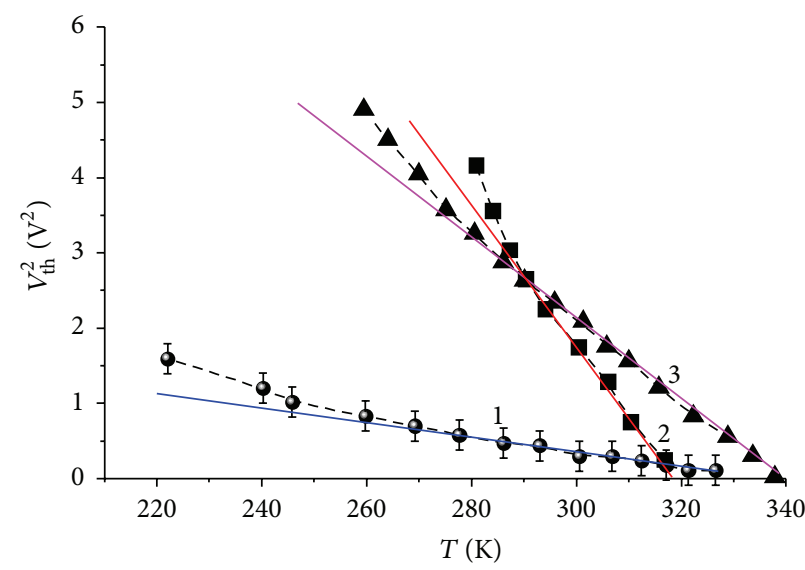

(a)

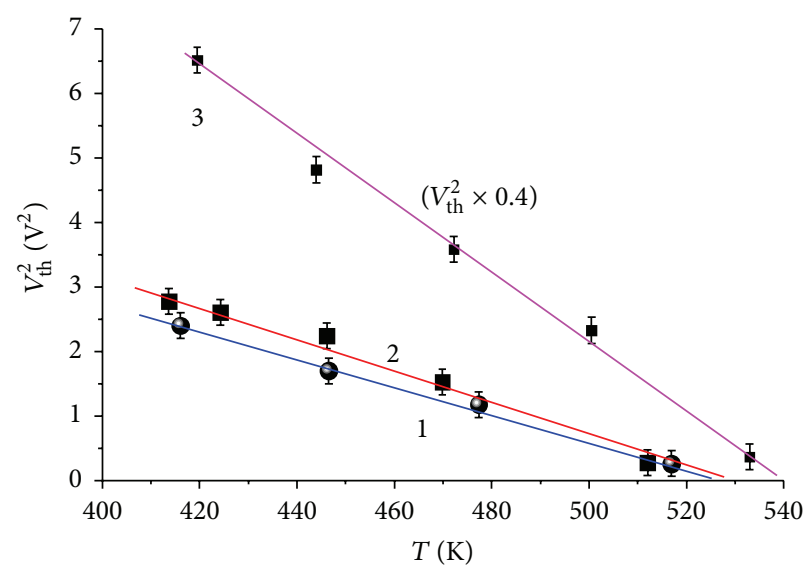

(b)

FIGURE 8: Squared threshold voltage as a function of temperature for (a) vanadium oxide-based switch and (b) titanium oxide; (1), (2), and (3): three different samples.

$T<340 \mathrm{~K}$ is a semiconductor, at $T=T_{t}=340 \mathrm{~K}$ the conductivity abruptly increases by $4-5$ orders of magnitude, and above the transition temperature $\mathrm{VO}_{2}$ exhibits metallic properties. For $\mathrm{NbO}_{2}, T_{t}=1070 \mathrm{~K}, \mathrm{Ti}_{2} \mathrm{O}_{3}$ undergoes a gradual transition in the range $400-600 \mathrm{~K}$, and magnetite $\left(\mathrm{Fe}_{3} \mathrm{O}_{4}\right)$ is a semiconductor both above and below the Verwey transition temperature $(120 \mathrm{~K})$, though its resistivity decreases on heating by two orders of magnitude at $T=T_{t}$. For the switching devices based on these metals, the temperatures $T_{o}$ coincide with the corresponding values of $T_{t}$ (see Figure 5 ). This suggests that the switching mechanism is caused by the metal-insulator transition.

Electrical switching due to MIT has been first revealed for vanadium dioxide $[16,27,37-45]$. Switching in $\mathrm{VO}_{2}$ is explained by the current-induced Joule heating of the sample up to $T=T_{t}$, which has been confirmed by the direct IR-radiation measurements of the switching channel temperature $[38,45]$. In these early works, switching has been observed in single crystals [39] and planar thin film devices [37, 38, 45], as well as in vanadate glasses [40, 41], $\mathrm{VO}_{2}$-containing ceramics [44], and $\mathrm{V}_{2} \mathrm{O}_{5}$-gel films [42, 43]. When as-prepared samples do not consist of pure vanadium dioxide, preliminary electroforming is required, resulting in the formation of the $\mathrm{VO}_{2}$-containing channel [40, 42]. The latter examples, as well as the discussed AOFs here, support the assumption that the formation of vanadium dioxide is thermodynamically preferable at a $\mathrm{V}_{2} \mathrm{O}_{5}-\mathrm{V}$ interface [22, 27]. For such samples, the threshold voltage decreases with temperature and reaches zero at $T=T_{t} \sim 340 \mathrm{~K}$.

In this case, the switching mechanism might be described in terms of the "critical temperature" model $[29,37]$ (if the ambient temperature is not much lower than $T_{t}$ ):

$$
V_{\mathrm{th}} \sim\left(T-T_{t}\right)^{1 / 2}
$$

that is, the squared threshold voltage linearly tends to zero at $T \rightarrow T_{t}$; see Figure 8 . Switching elements for other transition metal (Fe, $\mathrm{W}, \mathrm{Ti}$, and $\mathrm{Nb}$ ) oxides exhibiting MIT are expected to show similar behaviour, but for different $T_{t}$ 's which is demonstrated by Figure 8(b) for Ti oxide.

The switching effect in the $\mathrm{WO}_{3}$-based sandwich structures can also be explained in terms of a metal-insulator transition, which occurs in nonstoichiometric $\mathrm{WO}_{3-y}$ (within a very narrow interval of variable $y$ ) at $T_{t}$ ranging from 160 to $280 \mathrm{~K}$ [46]. Because of the narrow interval of "appropriate" stoichiometric compositions, the S-type $I-V$ characteristic is not likely to appear in tungsten trioxide, and in most cases breakdown occurs instead. For switching structures on anodic films on $\mathrm{W}, T_{o}$ was found to lie around $\sim 200 \mathrm{~K}$ (Figure 5). Therefore, $T_{o}$ may turn out to be equal to $T_{t}$. Almost no data on MIT in molybdenum oxides are available in the literature except for the transition in $\mathrm{Mo}_{8} \mathrm{O}_{23}$ $\left(\mathrm{MoO}_{2.875}\right)$, and the switching effect in molybdenum oxide will be discussed in more detail in the next section. As to $\mathrm{Ta}$ and $\mathrm{Hf}$ oxides, the formation of metastable lower oxides during electroforming should also be possible. The properties of these oxides are practically unknown, but some metastable oxides of this type have been referred to, for example, for the Ta-O system [47]. Recently, formation of $\mathrm{HfO}_{x}$ [48] and $\mathrm{TaO}_{x}$ [49] suboxides has been reported. As to $\mathrm{Mn}$ oxides, it is known [23] that $\mathrm{MnO}$ and $\mathrm{Mn}_{3} \mathrm{O}_{4}$ are Mott insulators, $\mathrm{Mn}_{2} \mathrm{O}_{3}$ is a semiconductor, and $\mathrm{MnO}_{2}$ is also a semiconductor, but it shows a change in electrical conductivity at $T=T_{N} \sim 90 \mathrm{~K}$ (the Neel temperature). This might account for the N-NDR effect in Mn oxide; this issue will also be more thoroughly investigated in the next section.

Thus, the experimental data on the switching effect in $d$-metal oxide thin films can be explained in terms of the switching mechanism driven by the MIT. We can state that in all cases the electrothermal and electrochemical processes during electroforming are responsible for the switching channel formation. The channel consists of a material which can undergo a transition from semiconducting state to metallic state at a certain temperature $T_{t}$. For the $\mathrm{V}$-, $\mathrm{Ti}$-, $\mathrm{Nb}$-, and $\mathrm{Fe}-$ based structures, the possibility of formation of the channels, consisting completely or partly of $\mathrm{VO}_{2}, \mathrm{Ti}_{2} \mathrm{O}_{3}, \mathrm{NbO}_{2}$, and 
$\mathrm{Fe}_{3} \mathrm{O}_{4}$, respectively, is also confirmed by the thermodynamic calculations. In oxides of $\mathrm{W}$ and Mo, switching may be associated with MIT in the channels consisting of $\mathrm{WO}_{3-x}$ and $\mathrm{Mo}_{8} \mathrm{O}_{23}$. The S-shaped voltage-current characteristic is conditioned by the development of an electrothermal instability in the channel. When a voltage is applied, the channel is heated up to $T=T_{t}$ (at $V=V_{\text {th }}$ ) by the current and the structure undergoes a transition from high-resistance $(\mathrm{OFF})$ insulating state to low-resistance $(\mathrm{ON})$ metallic state.

In high electric fields $\left(10^{5}-10^{6} \mathrm{~V} / \mathrm{cm}\right)$, the possible influence of electronic effects on the MIT should be taken into account. A field-induced increase in charge carrier density will act to screen Coulomb interactions [12], leading to the elimination of the Mott-Hubbard energy gap at $T<T_{t}$. Moreover, a transition of this type may be important in those materials where the usual temperature-induced MIT with a well-defined $T_{t}$ does not take place, for example, in oxides of Ta or Hf (and in CGS-based switching structures as well [50]). In the case of vanadium dioxide, such a possibility of the electronically induced MIT was suggested from the injection experiments [22] and will be discussed also below, in connection with the transition mechanism in $\mathrm{VO}_{2}$.

To summarize, the results presented above, as well as the other data on the switching in transition metal oxides [11-17, 27-31], indicate that current instabilities with the Stype NDR exhibit several common features. In particular, for each of the investigated materials there is a certain fixed temperature $T_{o}$, above which switching disappears. At $T<$ $T_{o}$, the threshold voltage decreases as the temperature rises, tending to zero at $T=T_{o}$. Comparison of these temperatures with the temperatures of MIT for some compounds $\left(T_{t}=\right.$ $120 \mathrm{~K}$ for $\mathrm{Fe}_{3} \mathrm{O}_{4}, \sim 200 \mathrm{~K}$ for $\mathrm{WO}_{3-x}, 340 \mathrm{~K}$ for $\mathrm{VO}_{2}, \sim 500 \mathrm{~K}$ for $\mathrm{Ti}_{2} \mathrm{O}_{3}$, and $1070 \mathrm{~K}$ for $\mathrm{NbO}_{2}$ ) shows that the switching effect is associated with the insulator-metal transition in an electric field. The channels, consisting of these lower oxides, are formed in initial anodic films during the process of preliminary EF.

And finally, in conclusion of this section, we would like to discuss one more vanadium oxide where the switching effects are caused by MITs, namely, vanadium sesquioxide. The $I-V$ characteristics of chromium-doped $\mathrm{V}_{2} \mathrm{O}_{3}$ shown in Figure 9 are distinctive in that they exhibit, in a certain temperature range, two regions of negative differential resistance: both $\mathrm{N}$ NDR and S-NDR. The experimental data on the switching effect of MOM structures based on $\mathrm{V}_{2} \mathrm{O}_{3}: \mathrm{Cr}$ can be explained in terms of the switching mechanism driven by the variable MIT. The S-shaped I-V characteristic is conditioned by the development of an electrothermal instability in the sample. When a voltage is applied, the sample is heated by the current up to $T=T_{t 1}$ (at the threshold voltage $V=V_{\mathrm{th}, S}$ ), and the structure undergoes a transition from a high-resistance (OFF-1) AFI state to a low-resistance (ON) PM state. As the current in the ON state increases, the sample is further heated, and at $T=T_{t 2}\left(I=I_{\mathrm{th}, N}\right)$ the second transition to the OFF-2 (or PI) state occurs [51]. In the OFF-1 state, the plot of current versus the square root of the applied voltage is indicative of a Poole-Frenkel type of conductivity enhancement [52] (Figure 10).

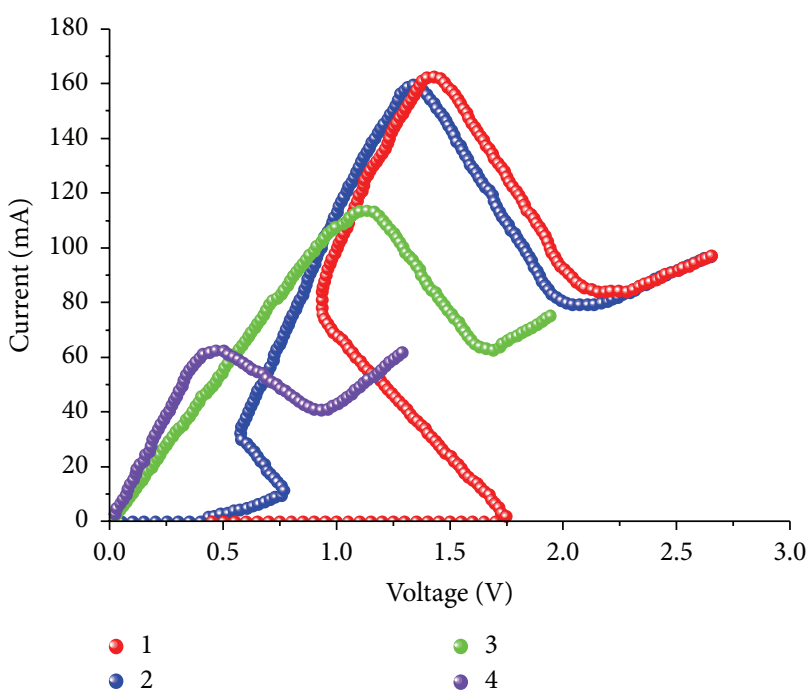

FIGURE 9: Current-voltage characteristics for $\mathrm{V}_{2} \mathrm{O}_{3}$ doped with 1.2 at.\% of $\mathrm{Cr}$ at different temperatures: $170.5 \mathrm{~K}$ (1), $173.3 \mathrm{~K}(2), 221.0 \mathrm{~K}$ (3), and $265.5 \mathrm{~K}$ (4) [51].

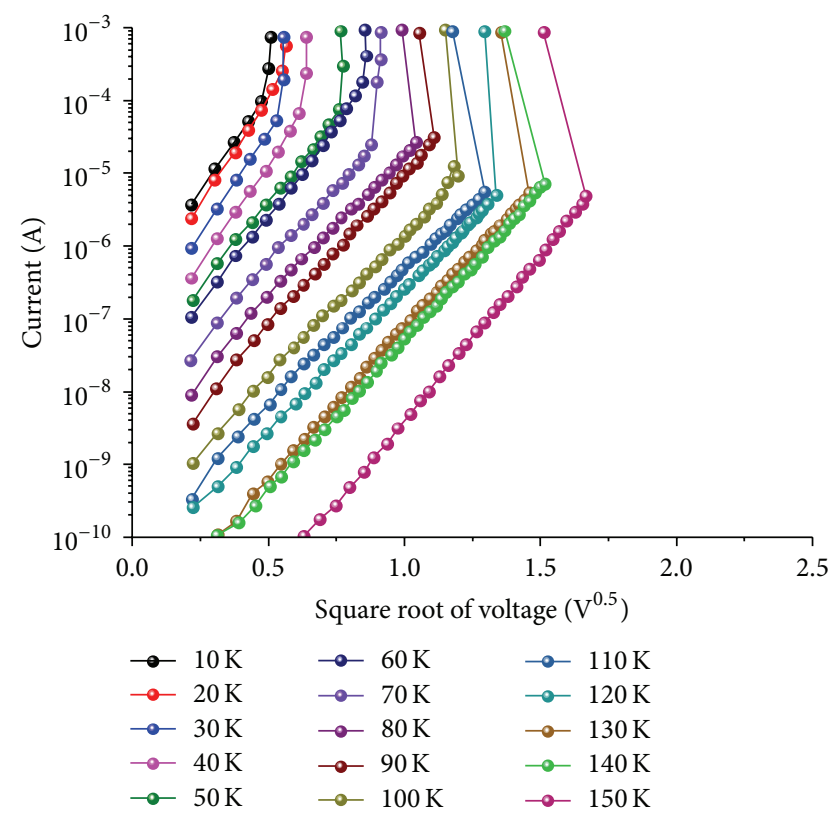

FIgURE 10: Current versus voltage in Poole-Frenkel coordinates for a $\mathrm{V}_{2} \mathrm{O}_{3}$-based switching device [52].

2.3. Switching Effects and Metal-Insulator Transitions in Mn and Mo Oxides. In this section, we consider electrical switching in oxides of $\mathrm{Mn}$ and Mo obtained by vacuum deposition, in comparison with those obtained by anodic oxidation [53, 54]. It should be emphasized here that AOFs are (as a rule, except for some rare cases) amorphous $[32,33]$. Therefore, in MOM structures with anodic films, the process of EF similar to that in CGSs usually takes place. However, in some cases (Mo, Mn), as was described in previous sections, we have failed to obtain stable switching with anodic films, and here we present an alternative approach to this problem. 
2.3.1. Electrical Switching in Thin Film Structures Based on Molybdenum Oxide. As in most TMOs, in the molybdenumoxygen system, there exist a number of oxides with variable valence which are characterized by a wide diversity of structures and physical properties $[53,55,56]$. Molybdenum trioxide $\mathrm{MoO}_{3}$ has a structure based on layers of edge-sharing distorted $\mathrm{MoO}_{6}$ octahedra [23]. Similar layered structures are inherent in lower molybdenum oxides, including $\mathrm{MoO}_{2}$, $\mathrm{Mo}_{18} \mathrm{O}_{52}, \mathrm{Mo}_{9} \mathrm{O}_{26}, \mathrm{Mo}_{8} \mathrm{O}_{23}, \mathrm{Mo}_{5} \mathrm{O}_{14}$, and $\mathrm{Mo}_{17} \mathrm{O}_{47}$, as well as $\eta$ - and $\gamma-\mathrm{Mo}_{4} \mathrm{O}_{11}$ [55-57].

Stoichiometric $\mathrm{MoO}_{3}$, in its most thermodynamically stable form $\alpha-\mathrm{MoO}_{3}$, is an insulator with a band gap of $\sim 3 \mathrm{eV}$ [23]. During reduction by means of either oxygen deficiency formation or introducing donor impurity atoms, additional donor levels near the conduction band bottom appear and the reduced oxide behaves therefore as a semiconductor. Polycrystalline $\mathrm{MoO}_{3}$, as well as a single crystal, has a greyyellow colour and the minimum value of the absorption edge is $440 \mathrm{~nm}$ which corresponds to the smallest value of the band gap of $2.8 \mathrm{eV}$ [58]; thus, the band gap depends upon the stoichiometry and the crystalline state [53]. The conductivity values of molybdenum oxides range from dielectric $\mathrm{MoO}_{3}$ to semiconductor $\mathrm{Mo}_{18} \mathrm{O}_{52}$ (resistivity $\rho=78.1 \Omega \cdot \mathrm{cm}$ ) and down to metal $\eta-\mathrm{Mo}_{4} \mathrm{O}_{11}\left(\rho=1.66 \times 10^{-4} \Omega \cdot \mathrm{cm}\right)$ [58]. Molybdenum dioxide also exhibits metallic properties [23, 58], and at room temperature $\rho=8.8 \times 10^{-5} \Omega \cdot \mathrm{cm}$ for bulk $\mathrm{MoO}_{2}$ samples [55].

Because of their special physical and chemical properties, molybdenum oxides are used in various electronic devices such as electrochromic displays and indicators, reversible cathodes of lithium batteries, gas sensors, and memory elements [55-61]. Metal-insulator transitions have also been observed in some oxides of molybdenum and related compounds [62-70]. In particular, $\mathrm{Mo}_{8} \mathrm{O}_{23}$ exhibits a two-stage structural MIT with formation of a charge-density wave at $T_{t 1}=315 \mathrm{~K}$ and $T_{t 2}=285 \mathrm{~K}$ [62]. However, as far as we know, no data are available in the literature concerning threshold switching associated with these transitions, except for our work [53]. In this work, we have particularly noted that memory switching is observed in many TMOs, molybdenum oxides included $[4,9,59,60]$.

The most discussed models in the literature for the ReRAM mechanism in oxide structures are those based either on the growth and rupture of a metal filament inside the oxide matrix under the action of electric current, or on the redox processes responsible for the formation of some high-conductivity or low-conductivity local inclusions corresponding to particular oxygen stoichiometry [4]. The MIT ideology is also sometimes involved to explain the properties of the structures and the memory switching mechanism therein [71]. In any case, the memory switching phenomenon seems to be associated with the ion transport [4,9]. It is also appropriate to mention here the works discussing the memory effects in a material with MIT (vanadium dioxide) associated with the presence of hysteresis in the temperature dependence of conductivity [72].

On the other hand, as was shown in the previous section, monostable threshold switching (with no memory effects) associated with the MIT is also characteristic of a number of TMOs. That is why the study of threshold switching in molybdenum oxide and its possible connection with the phenomenon of MIT is of considerable scientific and practical interest.

Thin film samples of molybdenum oxide were obtained by two ways: anodic oxidation and thermal vacuum evaporation [53]. Anodization of molybdenum was carried out in an acetone-based electrolyte ( $22 \mathrm{~g}$ of benzoic acid plus $40 \mathrm{~mL}$ of saturated borax aqueous solution per litre of acetone $[33,73])$. AOFs on Mo might include lower molybdenum oxides, for example, $\mathrm{Mo}_{4} \mathrm{O}_{11}$ and $\mathrm{MoO}_{2}$ with a relatively high electronic conductivity [53]. The fact that the anodic oxide on molybdenum contained lower oxides was confirmed by experimental data in the work [74], where it was shown that the degree of oxidation (valence state) of molybdenum in an $\mathrm{AOF}$ is less than six. Also, this was confirmed theoretically in terms of the thermodynamics of the process: the formation of unsaturated oxide $\mathrm{MoO}_{2}$ had been shown to be energetically more favourable than $\mathrm{MoO}_{3}$ [75].

Vacuum deposited film samples were fabricated by thermal evaporation of $\mathrm{MoO}_{3}$ powder at residual pressure of $10^{-4}$ Torr onto polished tantalum plates or glass substrates, for electrical and optical studies, respectively. The thicknesses of the films were determined from the transmission and reflection interference spectra measurements [76] and they were found to lie in the range from 300 to $800 \mathrm{~nm}$.

The XRD pattern of a vacuum-deposited molybdenum oxide sample is presented in Figure 11(a). It is shown that the samples represent amorphous $\mathrm{MoO}_{3}$ with a slight oxygen nonstoichiometry [53]. Anodic oxide films on Mo are also amorphous (or amorphous-microcrystalline, according to the literature data [73]), but their composition corresponds, as described above, to rather $\mathrm{MoO}_{2}$ or other lower oxides, and not to the highest oxide $\mathrm{MoO}_{3}$.

Figure 11(b) shows the reflection and transmission spectra for one of the samples obtained by vacuum deposition. The experimental data processing using the envelope of the interference extrema [76] yields the film thickness of (620 \pm $50) \mathrm{nm}$, and the absorption edge around $\lambda \approx 400 \mathrm{~nm}$ indicates the fact that the oxide phase composition corresponds to $\mathrm{MoO}_{3}$ with a band gap of $\sim 3 \mathrm{eV}$.

Before electroforming, initial structures demonstrate the nonlinear and slightly asymmetric current-voltage characteristics with no NDR regions. When the amplitude of the applied voltage reaches the forming voltage, a sharp and irreversible increase in conductivity is observed and the $I(V)$ curve becomes S-shaped (Figure 12). With increasing current, the $I-V$ characteristic may change until the parameters of the switching structure are finally stabilized. The process outlined above is qualitatively similar to the electroforming of the switching devices based on other TMOs (see Section 2.2 above). We emphasize once again that the phase composition of the ensuing switching channel must differ from the material of the pristine oxide film, because the channel conductivity is higher than that of the unformed structure by several orders of magnitude.

The current-voltage characteristics of vacuum-deposited films and those of anodic films are qualitatively similar, but 


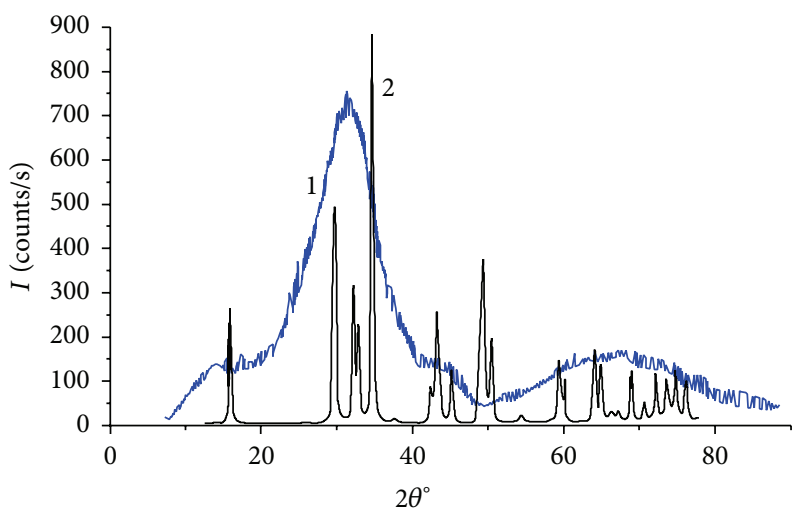

(a)

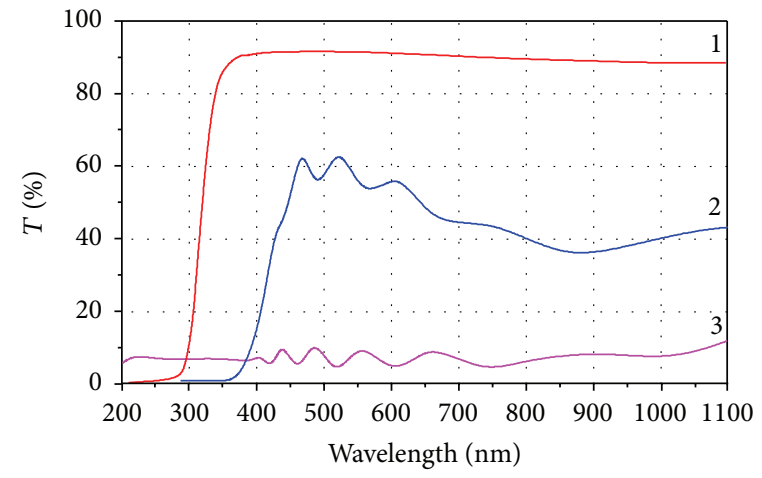

(b)

FIGURE 11: (a) XRD pattern of the molybdenum oxide film (1) and $\mathrm{MoO}_{3}$ powder (2). (b) Optical spectra of the sample obtained by vacuum deposition: (1) transmission coefficient of substrate (glass), (2) transmittance, and (3) reflectance of Mo oxide film on glass [53].

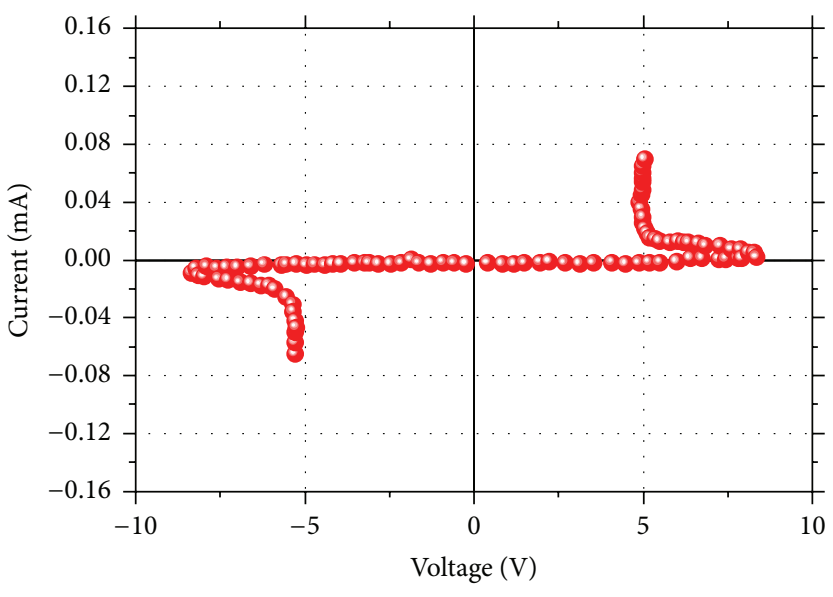

(a)

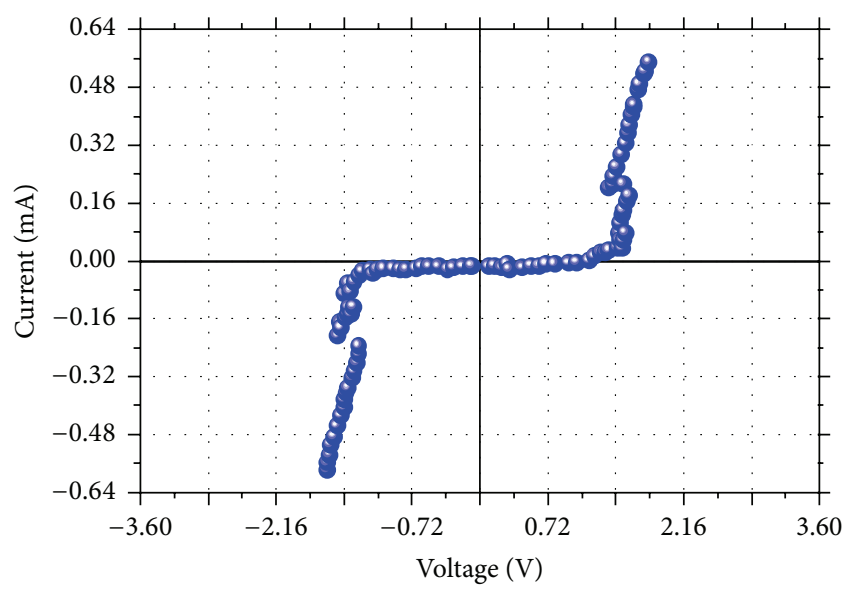

(b)

FIGURE 12: Current-voltage characteristics of MOM structures with vacuum-deposited (a) and anodic (b) Mo oxide films after electroforming.

there is a significant quantitative difference in their threshold parameters. For instance, the switching voltage $V_{\text {th }}$ for the AOF based structures is $\sim 2 \mathrm{~V}$ (Figure 12(b)), whereas for the structures on the basis of the films prepared by thermal evaporation $V_{\text {th }}$ is $\sim 10 \mathrm{~V}$ (Figure 12(a)). This difference can be explained by the difference in thickness of the films.

For anodic oxide, the thickness can be estimated from Faraday's law [53]:

$$
d=\frac{\mu I_{a} t}{4 e \rho S N_{A}},
$$

where $\mu=128 \mathrm{~g} / \mathrm{mol}$ and $\rho=6.5 \mathrm{~g} / \mathrm{cm}^{3}$ are, respectively, the molar mass and density of $\mathrm{MoO}_{2}$ and $e$ and $N_{A}$ are the unit charge and Avogadro constant. For $I_{a}=70 \mathrm{~mA}$ and $t=$ 10 min., (4) yields $d \sim 20 \mathrm{~nm}$ which is significantly less than the typical thickness of the vacuum deposited films and, as a result, the threshold voltage for AOFs is also lower than that for the films obtained by thermal evaporation.

As is discussed in [53], switching is not observed in thinner films obtained by vacuum evaporation $(d<100 \mathrm{~nm})$ in contrast to ultrathin AOFs. A plausible reason for this might be the fact that relatively thin vacuum deposited films contain through-the-thickness conducting defects due to local stoichiometry variations, whereas such defects are impossible in anodic films because they are grown in high electric field during anodic oxidation, and an appearance of such defects would interrupt the film growth process. This is similar to what is observed in vanadium oxide: in polycrystalline vanadium dioxide films such pin-hole defects are usually associated with the grain boundaries which could undergo local metallization due to strain or nonstoichiometry. That is why it had been difficult to observe switching in sandwich MOM structures with sputtered or thermally oxidized polycrystalline $\mathrm{VO}_{2}$ films and most studies had been conducted with planar thin-film structures, whilst for anodically oxidized $\mathrm{VO}_{2}$ the switching effect has then been observed in a sandwich structure for the first time in [16].

Next we note that, unlike the most other TMOs, the process of electroforming in Mo-based structures was hindered, as was mentioned above in Section 2.1; that is, the conventional electrical breakdown often occurred, not the 


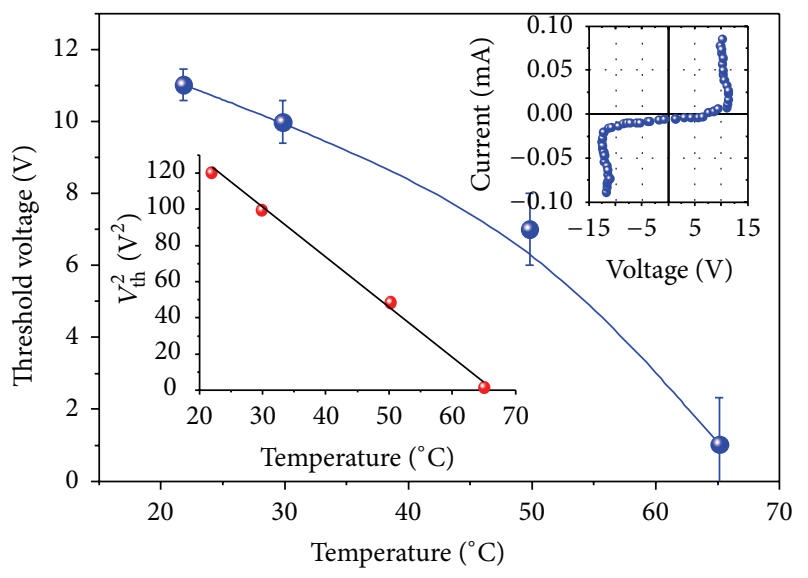

FIgURE 13: Threshold voltage as a function of temperature for the $\mathrm{Ta}-\mathrm{MoO}_{x}$-Au switching structure and the temperature dependence of $\left(V_{\mathrm{th}}\right)^{2}$ (lower inset) in compliance with (3). I- $V$ curve at RT (upper inset).

formation of a switching channel. However, for the Mo oxide films prepared by vacuum deposition, the processes of electroforming and switching were more stable which allowed in this case observation of the transformation of $I$ $V$ curves at a temperature change. It was found that, as the temperature increased, the threshold voltage was reduced. At $T=T_{o} \sim 55-65^{\circ} \mathrm{C}$, the NDR region degeneration commenced, at $T \approx 60-70^{\circ} \mathrm{C} V_{\text {th }} \rightarrow 0$, and at still higher temperatures, the switching effect no longer occurred. In this case, the switching mechanism might be described in terms of the "critical temperature" model (Figure 13). It is supposed [53] that, in the case of Mo, like in other TMObased structures, formation of a channel also takes place, and this channel should consist of a material exhibiting MIT, which is the reason for switching with an S-shaped currentvoltage characteristic (Figure 12).

A brief review of the materials with MIT in the family of molybdenum compounds presented in [53] shows that the most suitable candidate for the role of such a channel-forming compound is $\mathrm{Mo}_{8} \mathrm{O}_{23}$ because in this case the value of $T_{t 1}$ almost coincides with the above reported experimentally measured $T_{0}$. It should be noted that formation of other compounds of molybdenum (e.g., pyrochlores, bronzes, sulfides, or chalcogenide glasses), exhibiting MITs with similar order of magnitude transition temperatures $[64-68,70]$, is impossible because of the lack of the necessary chemical elements (i.e., rare earths, alkali metals or hydrogen, sulfur, and tellurium) in the phase composition of both the oxide film and the metal electrodes ( $\mathrm{Au}, \mathrm{Ta}$, and Mo).

In conclusion, the results presented in this subsection on switching from the HR state to the LR state with an S-NDR in molybdenum oxides obtained by both thermal vacuum deposition and electrochemical anodic oxidation show that the switching effect is due to, apparently, an insulator-tometal phase transition in the switching channel consisting, completely or partly, of the lower oxide $\mathrm{Mo}_{8} \mathrm{O}_{23}$, formed in the initial film during the process of electroforming. The current flowing through the channel heats it up to the MIT temperature $T_{t 1}=315 \mathrm{~K}[62]$ and the structure switches into the metallic low-resistance state.

Thus, the channels, consisting of this lower oxide, are formed in initial Mo oxide films during preliminary electroforming. Electrical forming results in the changes in oxygen stoichiometry conditioned by the ionic processes in an electric field close to the MOM structure breakdown field. This picture is quite similar to what is observed in many other TMOs exhibiting the insulator-to-metal transitions.

\subsubsection{Metal-Insulator Transition and Switching in Manganese} Dioxide. Manganese dioxide is one of the most interesting inorganic materials because of its wide range of applications. In particular, nanostructured $\mathrm{MnO}_{2}$ has received great attention due to its high specific surface area, functional properties, and potential applications as molecular and ion selective membranes, in capacitors, Li-ion batteries, and catalysts [77]. Originally, $\mathrm{MnO}_{2}$ has been widely used as a cathode material for oxide-semiconductor capacitors based on tantalum, niobium, and aluminium oxides, and the most important properties of the material for this application are its high conductivity and ability to undergo a thermal phase transition into the lower valence states accompanied by evolution of atomic oxygen, while the resistance increases by 3-4 orders of magnitude (see, e.g., [54] and references therein). Much attention is currently paid to various other properties of $\mathrm{MnO}_{2}$, such as, for example, the electrochromic effect [78], thermopower wave phenomenon [79], supercapacitive behaviour [80], and memory and threshold switching [54, 81, 82]. Also, there are a number of papers [83-85] describing an anomaly in the $\mathrm{MnO}_{2}$ conductivity near the Neel temperature which is shown to be associated with the metal-insulator transition [52]. That is why the electrical properties of $\mathrm{MnO}_{2}$ are of special scientific interest and applied importance.

The most common methods for manganese oxide preparation are the pyrolytic decomposition of manganese nitrate, electrochemical method, hydrothermal synthesis in a magnetic field, and chemical deposition [54, 86-89]. Due to a wide range of possible valence states of cations, the structure and phase composition of manganese oxides are determined by the synthesis conditions and process temperature. Manganese dioxide has many crystallographic forms $\left(\alpha-, \beta-, \gamma^{-}\right.$, $\delta-, \mathcal{E}^{-}$, and $\lambda-\mathrm{MnO}_{2}$ ) that connect $\mathrm{MnO}_{6}$ octahedron units to each other in different ways and have different characters of spatial alternation of the octahedra filled by $\mathrm{Mn}$ atoms and empty octahedra $[54,77,90-94]$.

The crystal structure of the $\beta-\mathrm{MnO}_{2}$ phase (Figure 14) is a tetragonal modification of the rutile structural type, $P 4 / \mathrm{mnm}$ symmetry group, which represents a slightly distorted closepacked hexagonal lattice of oxygen ions [93]. Filled octahedra are bound by two common edges in columns oriented along the $c$ axis, and octahedra of adjacent columns are bound by their vertices (Figure 14(c)). Manganese atoms are arranged in positions 2(a) (000), and oxygen atoms in positions $4(f)$ $(x x 0)$. Because there are vacancies in the oxygen packing, the formula unit can be written as $\mathrm{MnO}_{2-y}$ where the nonstoichiometry index is in the range $y=0.01-0.10$ depending on the synthesis method [93]. 


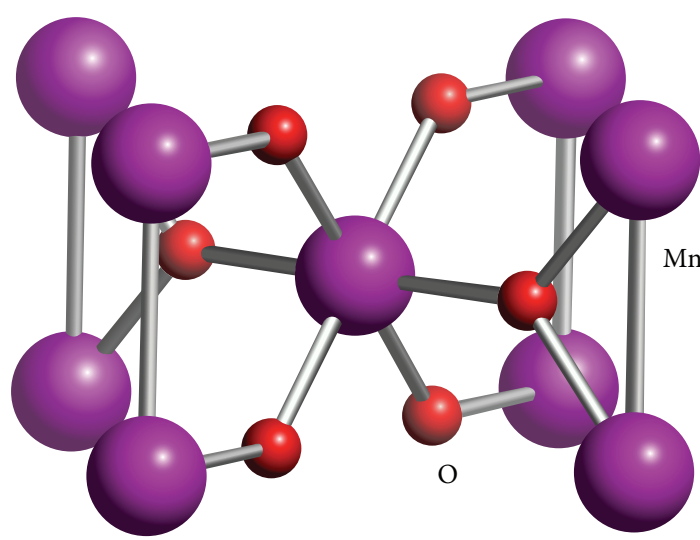

(a)

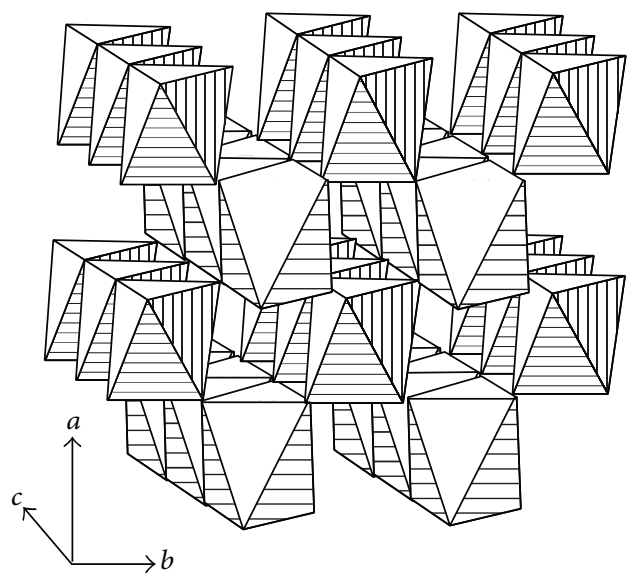

(b)

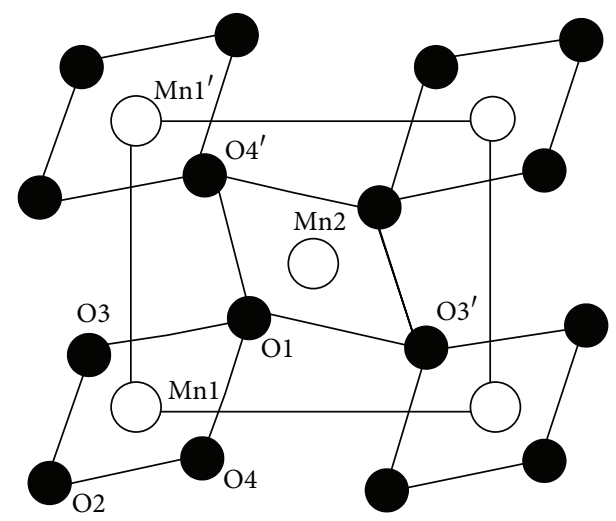

(c)

Figure 14: Crystal structure of manganese dioxide (a), $\mathrm{MnO}_{6}$ octahedron connection in $\beta-\mathrm{MnO}_{2}$ (b) [90-94], and projection of octahedron binding onto the $a b$ plane [93].

As to the lower manganese oxides, $\mathrm{MnO}$ and $\mathrm{Mn}_{3} \mathrm{O}_{4}$ are known to be the Mott insulators with relatively high resistivity [90], and $\mathrm{Mn}_{2} \mathrm{O}_{3}$ shows p-type semiconducting properties. $\mathrm{MnO}_{2}$ is an n-type semiconductor and antiferromagnetic with the Neel temperature $T_{N}=92.5 \mathrm{~K}$ [91]. Conductivity of manganese dioxide is higher than that of $\mathrm{MnO}$ and $\mathrm{Mn}_{2} \mathrm{O}_{3}$ by several orders of magnitude. At temperatures above $723 \mathrm{~K}$, manganese dioxide loses oxygen and transforms into $\mathrm{Mn}_{2} \mathrm{O}_{3}$, which is accompanied by the transformation of the crystalline structure from the tetragonal crystal system of the rutile type into the complex large-period cubic lattice $(a=9.4 \AA$ ) and a large number of formula units per unit cell $[54,92,93]$.

In this subsection, we present the results on electrical properties of manganese dioxide, particularly, the nature of the $\beta-\mathrm{MnO}_{2}$ conductivity at low temperatures, in its interrelation with characteristic features of the material crystalline structure, and the metal-insulator phase transition in this compound as well. The effect of electrical switching with current-voltage characteristics of both $\mathrm{N}$ - and S-type has been found in thin-film MOM structures on the basis of manganese oxides. Possible mechanisms of switching in terms of the MIT and thermistor effects are discussed.

Mn oxide samples under study have been obtained by three methods: anodic oxidation and vacuum evaporation (thin films), as well as by pyrolysis [54]. Thin films of manganese dioxide were deposited by means of thermal evaporation onto glass substrates, covered by a $\mathrm{SnO}_{2}$ layer, using a VUP-5 vacuum setup; the film thickness was $d=$ $250 \mathrm{~nm}$. Also, thin films of manganese oxide were obtained by electrochemical oxidation of metal $\mathrm{Mn}$ in the $\mathrm{KNO}_{3}-\mathrm{NaNO}_{3}$ eutectic melt (see Section 2.1) at $T=600 \mathrm{~K}$ at a constant voltage of about $2 \mathrm{~V}$ for $3 \mathrm{~min}$; the current density diminished from 200 to $100 \mathrm{~mA} / \mathrm{cm}^{2}$ during the oxidation process. For anodic oxide films on $\mathrm{Mn}$, the value of $d$ was $\sim 100 \mathrm{~nm}$. Bulk samples of manganese dioxide were obtained by multiple decomposition (pyrolysis) of manganese nitrate at $T=670 \mathrm{~K}$ on a glass-ceramic substrate or on a tantalum sheet metal with the subsequent separation from the substrate [92, 93]. The total coating thickness was about $1 \mathrm{~mm}$.

The manganese oxide samples were characterized by $\mathrm{X}$ ray diffraction (XRD) using a DRON-6 diffractometer in $\mathrm{CuK}_{\alpha}$ and $\mathrm{FeK}_{\alpha}$ radiation. The characteristics of the atomic structure of pyrolytic manganese dioxide were refined by the full-profile analysis of the XRD patterns of polycrystalline samples [93]. The resistivity of pyrolytic $\mathrm{MnO}_{2}$ was measured in the temperature range 15 to $300 \mathrm{~K}$ by means of a four-probe technique, both DC and AC (in a frequency range from 50 to $10^{4} \mathrm{~Hz}$ ), in a Gifford-McMahon cycle cryorefrigerator [54]. We also measured electrical conductivity in the range $T=$ $293-423 \mathrm{~K}$, the Hall effect in the magnetic field of $2.3 \mathrm{~T}$, and the Seebeck coefficient (thermoelectromotive force, thermoemf). The dynamic current-voltage $(I-V)$ characteristics of the thin-film MOM structures were measured by oscillographic method using an AC sinusoidal signal.

The XRD patterns of the manganese oxide samples are presented in Figures 15 and 16. The vacuum-deposited films are amorphous and represent a mixture of $\beta$ - and $\gamma-\mathrm{MnO}_{2}$ (Figure 16). The results of the full-profile analysis show that the pyrolytic $\mathrm{MnO}_{2}$ samples (Figure 15) are composed of the finely dispersed $\beta-\mathrm{MnO}_{2}$ phase with the traces of $\gamma$ $\mathrm{MnO}_{2}, \varepsilon-\mathrm{MnO}_{2}$, and $\mathrm{Mn}_{2} \mathrm{O}_{3}$ phases. The periods of the tetragonal unit cell of $\beta-\mathrm{MnO}_{2}$ are $a=b=4.401 \AA$ and $c=2.872 \AA$. The oxygen coordinates in $4 f$ positions (see discussion of the crystal structure above and Figure 14) are found to be $x=0.302$. Calculations of the oxygen-manganese interatomic distances show [93] that the oxygen octahedron 


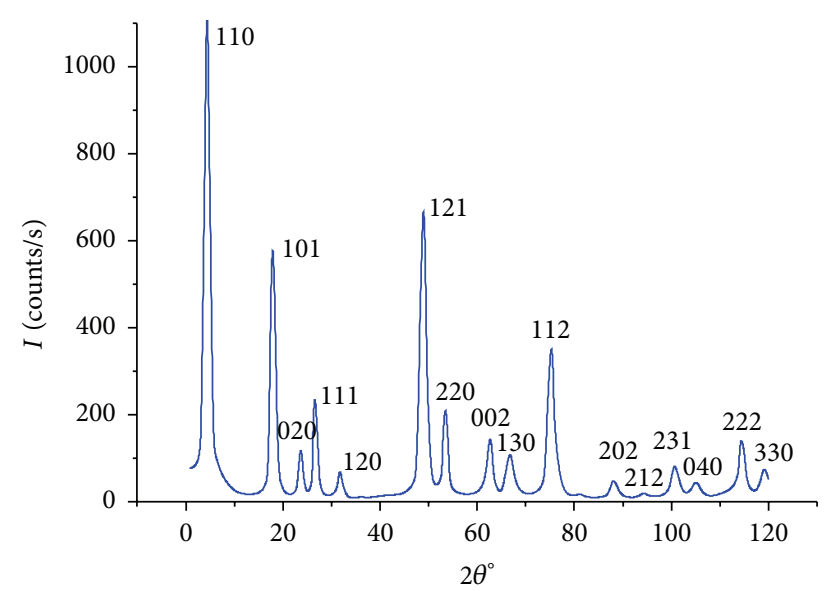

FIGURE 15: XRD (Cu K ${ }_{\alpha}$ radiation) pattern of pyrolytic $\mathrm{MnO}_{2}$.

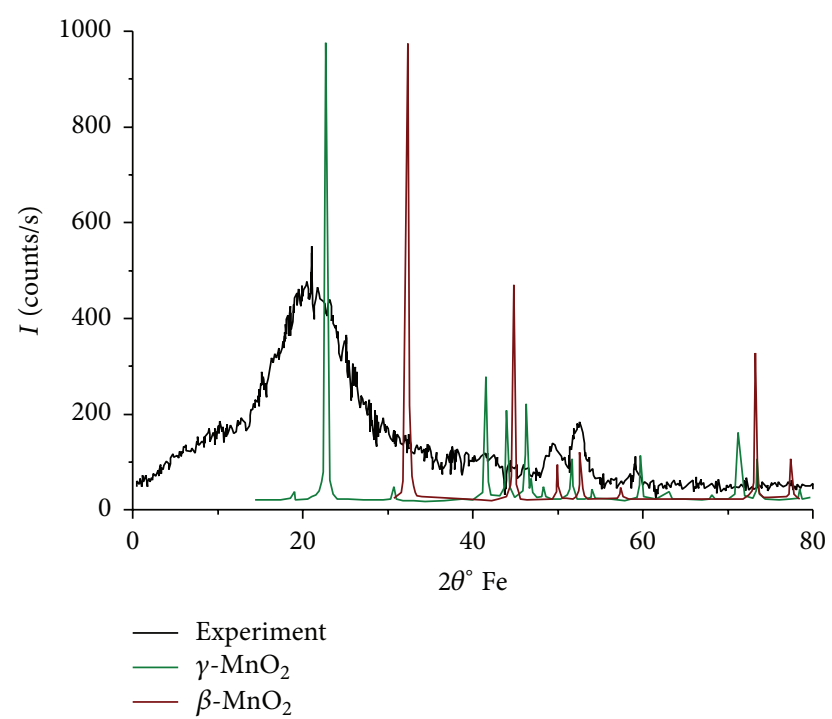

FIGURE 16: XRD (Fe $\mathrm{K}_{\alpha}$ radiation) pattern of Mn oxide film and reference spectra of crystalline $\mathrm{MnO}_{2}$ samples.

is compressed along the (100) direction which connects the octahedron vertices. This deformation results in an increase of the overlap of electron $p$ orbitals of oxygen and $d$ orbitals of manganese and can form the additional splitting of electron levels in the crystal field leading to the appearance of unpaired electrons [94].

The measurements of the conductivity temperature dependence, Hall effect, and thermo-emf show that manganese dioxide is the electron semiconductor with conductivity of about $1 \Omega^{-1} \mathrm{~cm}^{-1}$ and mobility of $\sim 10 \mathrm{~cm}^{2} / \mathrm{V} \cdot \mathrm{s}$. The Hall measurements give the carrier density of $\sim 10^{18} \mathrm{~cm}^{-3}$, which is close to the density values in degenerate semiconductors. The activation energy of conductivity is $\sim 0.1 \mathrm{eV}$, and the band gap width is equal to $2 \mathrm{eV}$ [90]. The estimated value of the Fermi energy, from the thermo-emf temperature dependence, is in the range of 0.02 to $0.05 \mathrm{eV}$ which also indicates the degenerate character of the electron gas. The nonstoichiometry index determined by the XRD peaks, which are not contributed by the manganese atoms, that is, by those with the odd sum of indexes, is $y=0.06$. Such nonstoichiometry provides the concentration of trap states of about $10^{17}-10^{18} \mathrm{~cm}^{-3}$, which correlates with the above mentioned Hall-effect-based measurements of electron density.

Such high vacancy concentration allows us to consider the nonstoichiometric $\beta-\mathrm{MnO}_{2}$ as a heavily doped semiconductor with a subband of impurity levels, which provides a feasibility of the out-of-band electron transfer at low temperatures. The structural distortions, high nonstoichiometry and, as a consequence, sufficiently high concentration of impurity states, cause the density state tails near the band edges similarly to those in heavily doped semiconductors. However, at room and higher temperatures the conduction is determined by the activation mechanism. Thus, the electron structure and a low mobility of charge carriers promote the manifestation of different charge transfer mechanisms in different temperature ranges, namely, both the hopping mechanism and the activation one.

The electron capturing oxygen vacancies create mixed valence states of manganese cations $\left(\mathrm{Mn}^{3+}-\mathrm{Mn}^{4+}\right)$. Interestingly, the mixed valence of $\mathrm{Mn}$ ions is also characteristic of manganites $\mathrm{Ln}_{1-y} \mathrm{Sr}_{y} \mathrm{MnO}_{3}$ [95], where $\mathrm{Ln}$ is $\mathrm{La}$ or another rare-earth element, possessing the effect of colossal magnetoresistance (CMR). In these compounds, the mixed valence of manganese is attained via doping. It had been deemed that holes introduced by strontium are localized at manganese ions; that is, the $\mathrm{Mn}^{4+}$ ions with concentration $y$ are formed. However, recent X-ray emission studies have shown that both the chemical shift and the exchange splitting of the emission $\mathrm{X}$-ray $\mathrm{Mn} \mathrm{K}_{\beta 1}$ line are almost the same in the range $y<0.4-$ 0.5 , as is the case in $\mathrm{Mn}_{2} \mathrm{O}_{3}$, and then they decrease quickly to the values characteristic of $\mathrm{MnO}_{2}$ [96] which suggests that the introduced holes are localized at oxygen atoms in the hole doping region (at relatively low $y$ ) and at manganese atoms in the electron doping region, that is, at higher $y$ values.

The temperature dependence of conductivity is presented in Figure 17. The resistance peak observed in the figure in a temperature range of $80-90 \mathrm{~K}$ is the result of the phase transition of $\beta-\mathrm{MnO}_{2}$ from a semiconducting state to a metal state upon decreasing the temperature to $15 \mathrm{~K}$ [54]. The temperature region of the transition is close to the known Neel transition from the paramagnetic state into the ferromagnetic one for manganese dioxide. The temperature dependence of resistance indicates thus the MIT to occur in $\mathrm{MnO}_{2}$. In the temperature range from 300 to $80 \mathrm{~K}$ (Figure 17), this dependence does not correspond to the exponential one, yet it fits well with the linear dependence in the Mott coordinates $\ln (R) \sim T^{1 / 4}$, which indicates the hopping conductivity mechanism over the polyvalent states of manganese ions with a distance between the centres of 25-35 $\AA$ [92]. However, in the temperature range from 90 to $15 \mathrm{~K}$, the dependence of $\ln R$ on $\ln T$ is almost linear with the slope of about unity (i.e., $\partial \ln R / \partial \ln T \approx 1$ ), which is characteristic of the metallic conduction. Thus, the character of conductivity of manganese dioxide indicates the presence of the MIT at $T_{c} \sim 80 \mathrm{~K}$ (on cooling). 


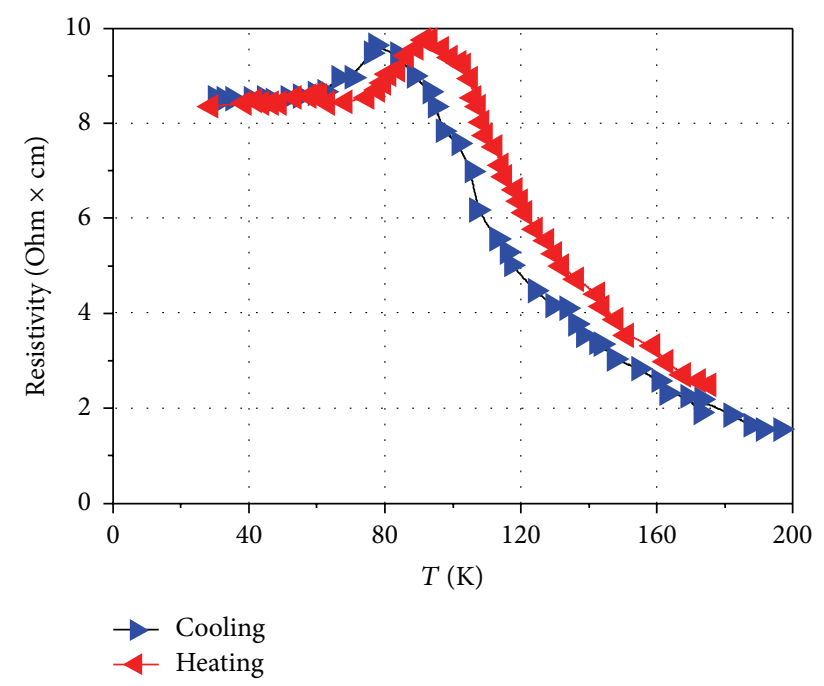

FIgURE 17: Temperature dependence of $\mathrm{AC}(70 \mathrm{~Hz})$ resistivity of pyrolytic $\mathrm{MnO}_{2}$.

In the low temperature region, manganese dioxide is an antiferromagnet with the helical spin ordering [97]. The interaction between the magnetic ions of manganese, which leads to the antiferromagnetic state, occurs through the intermediate interaction with diamagnetic oxygen ions, which weakens the exchange interaction. The conductivity increases probably due to exceeding the energy of the exchange interaction by the thermal energy as the temperature increases, which leads to an increase in the number of unpaired electrons in the paramagnetic state. As the temperature further increases, the conductivity continues to rise, and the Mott hopping mechanism of charge transfer becomes predominant. Similar character of the temperature dependence in ferromagnetic degenerate semiconductors has previously been described $[85,98]$ for substoichiometric (oxygen-deficient) europium monoxide. The transition from the highly conductive state into the insulating state occurs in such semiconductors upon varying the type of magnetic ordering or its destruction.

For $\mathrm{EuO}_{1-y}$, lowering the peak magnitude in the $R(T)$ dependence is associated with a decrease in the concentration of impurities (oxygen vacancies). In magnetic semiconductors, the phase transition is associated both with the magnetoelectric effects and with the variation in the electric polarization under the action of the magnetic field at the antiferromagnetic transition [98]. The dielectric constant for manganese dioxide is equal to $\sim 10$, but the effective dielectric constant can decrease near $T_{c}$ by a factor of several times thereby affecting the mobility of carriers located in the tails of the density of states near the band edge and promoting their delocalization at the phase transition temperature. Manganese dioxide also belongs to this group of substances; however, the phase transition in $\mathrm{MnO}_{2}$ reported here can be also described in terms of the Mott transition [24].

According to the Mott criterion, electrons delocalize if the distance between the atoms $n^{-1 / 3}$ becomes comparable with their atomic radius $a_{A}$, where $a_{A}$ is associated with the Bohr radius $a_{B}=\hbar^{2} \varepsilon / m e^{2}$. The static permittivity $\varepsilon$ is replaced by the effective permittivity, which takes into account the exchange interaction of the spins of conduction electrons with orbital magnetic moments of atoms. At the temperatureinduced Mott MIT, the orbital radius $a_{B}$ decreases near $T=$ $T_{c}$ thereby providing the fulfillment of the Mott criterion at a given level of impurity centres $[24,98]$. The transition from the metallic state into the semiconductor state is possible in such semiconductors as the temperature increases. Thus, the MIT in manganese dioxide is brought about by temperature variation.

In conclusion, we note that the phase transition described above is inverse (reentrant) MIT; that is, the metallic phase is low-temperature, while the high-temperature state is insulating. This is rather rare, yet not unique, and unusual phenomenon. In most cases (e.g., in vanadium dioxide), the low-temperature phase is insulating, and on heating above a certain temperature $T_{c}$, the material becomes metallic; for $\mathrm{VO}_{2}$, this transition temperature is $T_{c}=340 \mathrm{~K}$. The inverse transitions are observed in such compounds as the above mentioned $\mathrm{EuO}_{1-y}, \mathrm{NiS}_{2}: \mathrm{Se}, \mathrm{Y}_{2} \mathrm{O}_{3-y}$, and $\mathrm{LaMnO}_{3}$ films [24, $31,52,85,98,99]$. Also, the high-temperature Mott transition from paramagnetic metal to paramagnetic insulator in $\mathrm{V}_{2} \mathrm{O}_{3}$ : $\mathrm{Cr}$ is an inverse transition with the resistivity peak at $T_{c} \sim 350 \mathrm{~K}$ for a chromium concentration of around 1 at. $\%$ $[24,51]$, and in mixed valence CMR-manganites the sharp resistivity peak has been shown to be caused by the Anderson MIT [95].

It is known that many TMOs exhibit electrical switching associated with MITs [28]. For the anodic oxide films on $\mathrm{Mn}$, the study of the effect of electrical switching in thinfilm $\mathrm{Mn}-\mathrm{MnO}_{2}-\mathrm{Au}$ sandwich structures showed that the Stype switching was not observed in contrast to, for example, the similar structures based on oxides of vanadium and some other transition metals [28]. Instead, switching with the $\mathrm{N}$-shaped $I-V$ characteristic occurred after EF at $T<$ $80 \mathrm{~K}$ (Figure 18(a)). In addition, the EF process in the Mnbased structures was hindered, likewise, for example, for the structures based on yttrium oxide [31]; that is, the breakdown took place more often. It should be noted that EF at room temperature always led to breakdown, and the switching effect had never been observed in this case. As was argued in [54], this switching effect was associated with the MIT in $\mathrm{MnO}_{2}$.

On the other hand, in the films obtained by vacuum sputtering, we have observed S-type switching at room temperature (Figure 18(b)). The threshold voltage is of the order of $1 \mathrm{~V}$ and almost independent of temperature up to $T$ $=375 \mathrm{~K}$.

This situation resembles that with yttrium oxide where both S-type and N-type switching has been observed too [31]. One can assume that switching with the S-shaped $I-V$ curves is due to the so-called "thermistor effect." It is well known that the current flowing through a solid generates Joule heat, resulting in an increased temperature. In materials with a negative TCR, a rising temperature induces a current enhancement and an increased power dissipation resulting in a farther temperature increase. This positive electrothermal feedback causes the appearance of an NDR and, accordingly, an S-shaped $I-V$ characteristic [2]. 


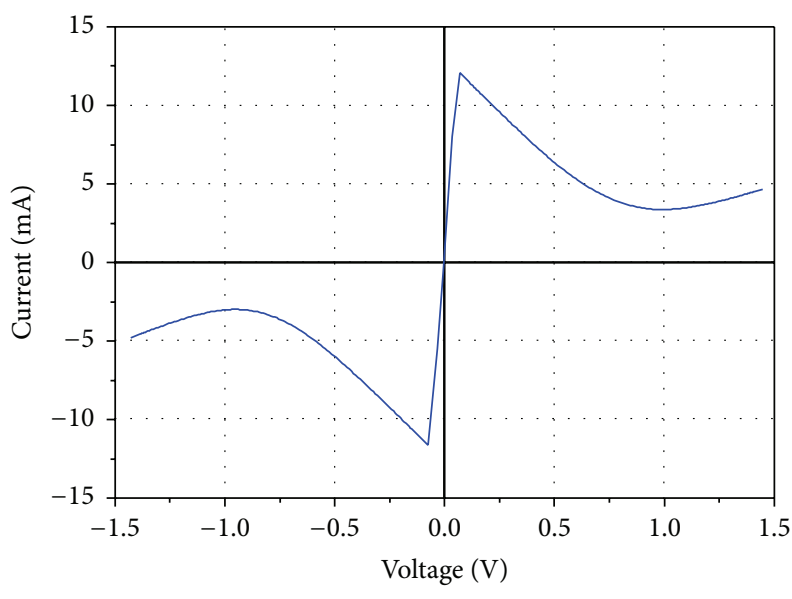

(a)

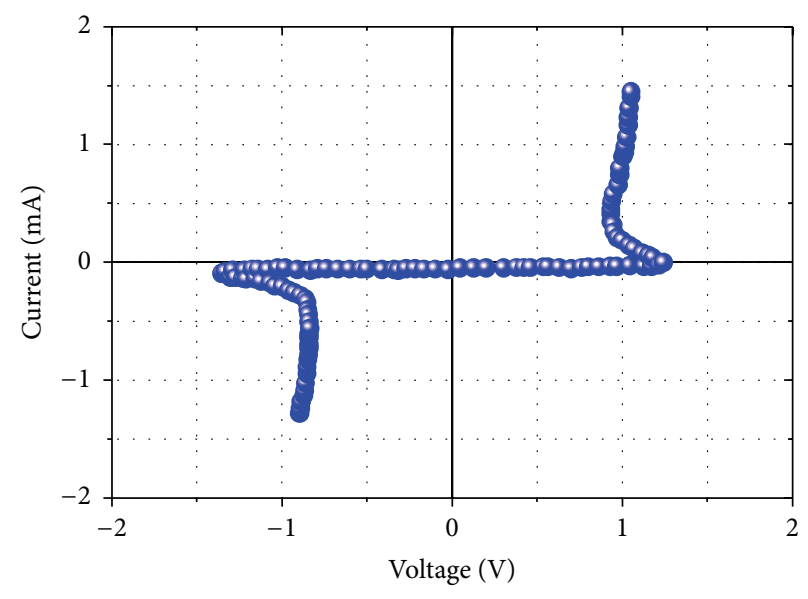

(b)

FIgURE 18: (a) $I-V$ characteristic of anodic oxide film on manganese at $T=77 \mathrm{~K}$. (b) $I-V$ characteristic of vacuum-deposited $\mathrm{MnO}_{2}$ at $T=$ $293 \mathrm{~K}$.

In some cases, this process might by modified by high-field effects, such as Schottky injection, impact ionization, PooleFrenkel effect, and Zener or avalanche breakdown. Note that, unlike a conventional thermistor with an Arrheniuslike conductivity law, manganese oxide exhibits a weaker $R(T)$ dependence characteristic of the hopping conductivity mechanism which seems to account for the observed weak temperature dependence of the threshold voltage.

An alternative mechanism for the S-type switching effect may be connected with a possible high-temperature usual (i.e., not inverse) MIT in some manganese oxide. As is known, EF (see Section 2.2) leads to the formation of a filament of highly conducting material, a switching channel. Similarly to what is observed for Mo oxide (Section 2.3.1) and other TMOs (Sections 2.1 and 2.2), we suppose that the composition of this channel differs from the material of the initial oxide film, since its conductivity noticeably exceeds that of an unformed structure. In view of such a plentiful set of phases and crystal structures in the $\mathrm{Mn}-\mathrm{O}$ system, one can suppose that the channel might consist of nonstoichiometric $\beta-\mathrm{MnO}_{2}$, some other polymorphic modifications of manganese dioxide, or lower Mn oxides, which might undergo a MIT under the action of either temperature or electric field. However, this question obviously requires additional investigation, in particular, measurements of $R(T)$ dependences and switching properties at higher temperatures. If this assumed hightemperature MIT would occur in, for example, nonstoichiometric $\beta-\mathrm{MnO}_{2}$, one could expect observation of doubleNDR I-V characteristics in such samples at low temperatures, analogously to those in $\mathrm{V}_{2} \mathrm{O}_{3}$ : $\mathrm{Cr}$ (Figure 9) where this effect is due to the MITs [29], with the only difference being that in the case of $\mathrm{MnO}_{2}$ this curve should be N-S-shaped (unlike the $\mathrm{S}-\mathrm{N}$-shaped one in Figure 9), since in chromium-doped vanadium sesquioxide the inverse MIT is high-temperature as opposed to $\mathrm{MnO}_{2}$ which, as we discussed above (see Figure 17), exhibits the inverse MIT at low temperatures.

Note however that the discussed high-temperature MIT in $\beta-\mathrm{MnO}_{2-y}$ may be unobservable under the temperature change in equilibrium conditions but displays itself only under the applied electric field as the S-type electrical switching effect, because the transition temperature is close to or higher than the temperature of $\mathrm{MnO}_{2}$ decomposition $(723 \mathrm{~K})$. Such a nonequilibrium transition occurs at switching in those materials where the usual temperature-induced MIT with a well-defined $T_{t}$ does not take place, for example, in CGSs for which the equilibrium transition temperature $T_{t 0}$ is higher than $T_{g}$, while in a high electric field $T_{t}(E)<T_{t 0}$ might be lower than $T_{g}$, where $T_{g}$ is the temperature of the glass-crystal transition and $E$ is the electric field strength [30].

Summarizing, we have studied the electrical properties (conductivity temperature dependence and Hall and Seebeck effects) of the $\beta$ phase of manganese dioxide obtained by pyrolysis, thermal vacuum evaporation, and anodic oxidation. The change of conductivity mechanisms depending on temperature in a range of $450-25 \mathrm{~K}$ has been shown and the metal-insulator phase transition at low $(<90 \mathrm{~K})$ temperatures has been revealed. The specific feature of this transition described in terms of the Mott mechanism is that the metal phase exists at low temperatures, while at higher temperatures the material is a semiconductor with the hopping conductivity over the mixed valence states of manganese ions.

The low-temperature switching effect with an NDR and $\mathrm{N}$-shaped current-voltage characteristic associated with the phase transition in manganese dioxide has been found in the MOM two-terminal devices based on anodic Mn oxide films. On the contrary, the devices based on vacuum-deposited films have been found to exhibit an S-NDR at room and higher temperatures which is suggested to be caused by either the thermistor effect or a high-temperature MIT in nonstoichiometric $\beta-\mathrm{MnO}_{2}$.

It should be emphasized that the low-temperature MIT in $\beta-\mathrm{MnO}_{2}$ with $T_{t}=70-80 \mathrm{~K}$ is the inverse Mott transition which is akin, for instance, to the transition in $\mathrm{V}_{2} \mathrm{O}_{3}: \mathrm{Cr}$ at $T_{t}=190-385 \mathrm{~K}$ (for chromium dopant concentration in the range of $0.5-1.8$ at.\%) [51]. We thus assume that farther 


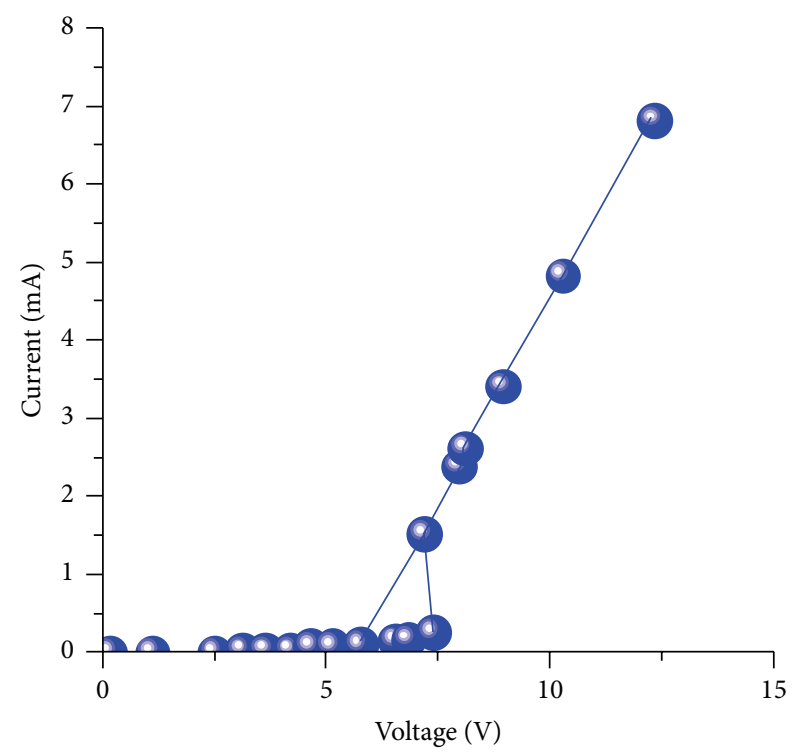

(a)

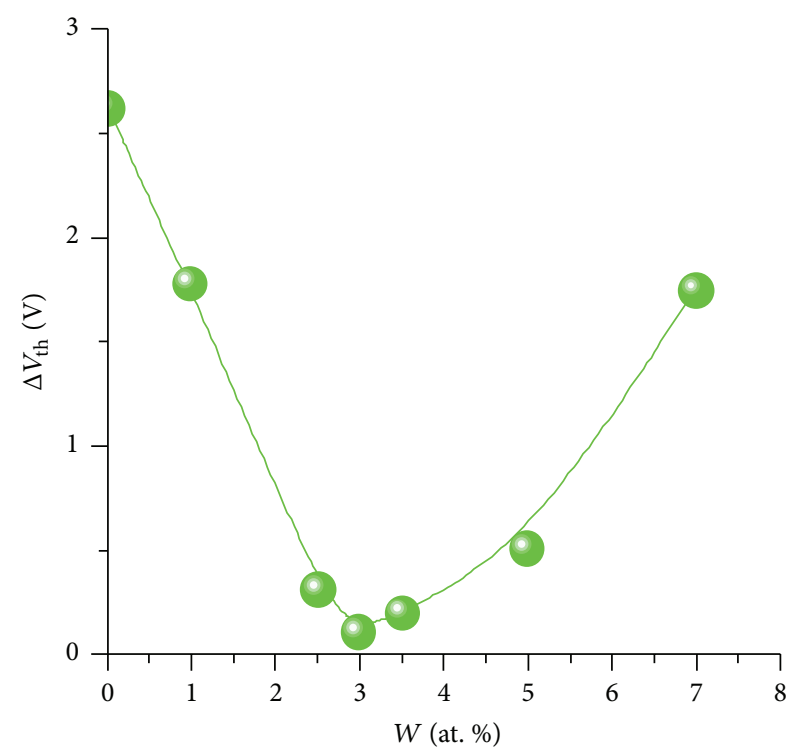

(b)

FIGURE 19: $I-V$ curve (a) of Au- $\mathrm{V}_{2-y} \mathrm{~W}_{y} \mathrm{O}_{5} \times n \mathrm{H}_{2} \mathrm{O}$-Au sandwich structure with $y=0.03$ and $V_{\text {th }}$ scattering as a function of tungsten admixture percentage $y$ (b) $[104,105]$.

investigations of the phase transition in manganese dioxide described in this paper are a rather interesting and topical scientific problem, since manganese dioxide can serve as a model object for studying such inverse metal-insulator phase transitions.

Finally, we note that the results presented in this section indicate the possibility of application of manganese oxide as a material for thin film sandwich switching devices. Devices with NDR (N- and S-shaped I-V characteristics) have potential applications in microelectronics as switches, memory elements, and microsensors (these issues will be discussed in more detail below, in Section 4). Particularly, manganese oxide microswitches [82] can be utilized for implementation of neuristors built with Mott-transition-based memristors [100].

2.4. Effect of Doping. It is only natural that the systems exhibiting the NDR effects possess a high applied potential in electronics $[2,4,8,18,100,101]$, yet the stage of $\mathrm{EF}$ is a weak link of chain in the technology process. Note that the forming process may be brought about by laser irradiation [102], not by applying of electric field. In the case of laser treatment (or "laser forming," unlike the above described electrical forming), the parameters of the structures are characterized by the absence of the scatter; that is, the $I-V$ characteristics of all structures are almost identical [103]. One more demerit to be avoided is the scatter in switching parameters (the value of $V_{\text {th }}$ first of all) and their instability during operation. In this subsection, we discuss the issues concerning the switching parameter stabilization by means of doping.

In the work [104], we have studied the switching effect in the MOM structures on the basis of $\mathrm{W}$-doped $\mathrm{V}_{2} \mathrm{O}_{5}$-gel films. These structures require preliminary EF, similar to that in anodic oxide films, resulting in formation of a conducting channel within the initial dielectric xerogel film. The channel consists of vanadium dioxide and switching is associated with the insulator-to-metal transition in $\mathrm{VO}_{2}$. As was shown in $[104,105]$, for the pure (undoped) $\mathrm{V}_{2} \mathrm{O}_{5}$-gel, the switching parameters vary in a wide range, because the phenomenon of EF is statistical in nature (like electrical breakdown), and its statistical character shows itself as a spread in the observed threshold voltages of the ensuing structures; that is, actually, at multiple measurements, $V_{\text {th }}=\left\langle V_{\text {th }}\right\rangle \pm \Delta V_{\text {th }}$.

It has been shown [104] that the statistical spread in values of the threshold parameters can be minimized by means of doping (Figure 19). Unsophisticated reasoning could account for this result: for pure $\mathrm{V}_{2} \mathrm{O}_{5}$-gel, electroforming leads to formation of $\mathrm{VO}_{2}$ with $T_{t}=340 \mathrm{~K}$, and each switching event requires heating up and cooling down from room temperature to $T_{t}$; this thermal cycling can modify the peripheral regions of the channel, which leads to the change of the switching threshold voltage. On the other hand, for the samples with a high concentration of W, the MIT is suppressed [104], and switching is degenerated. Between these two outermost points, an optimum should exist. As one can see from Figure 19, this optimal value corresponds to 3 at.\% of $\mathrm{W}$; that is, for $\mathrm{V}_{0.97} \mathrm{~W}_{0.03} \mathrm{O}_{2}$ the switching parameters are the most stable. The transition temperature of $\mathrm{V}_{0.97} \mathrm{~W}_{0.03} \mathrm{O}_{2}$ is lower than that for pure vanadium dioxide, and hence the magnitude of the aforesaid thermal cycling is decreased, which results in an increase of stability of the switching parameters.

Next we have studied the effect of doping on switching in AOFs on $\mathrm{Ti}$ [29]. These films were obtained in $5 \%$ water solution of citric acid $\left(\mathrm{C}_{6} \mathrm{H}_{8} \mathrm{O}_{7}\right)$, in volt-static regime at $V_{a}$ $=20 \mathrm{~V}$. The samples were doped with aluminium by means of adding soluble Al-containing substances to the electrolyte. 


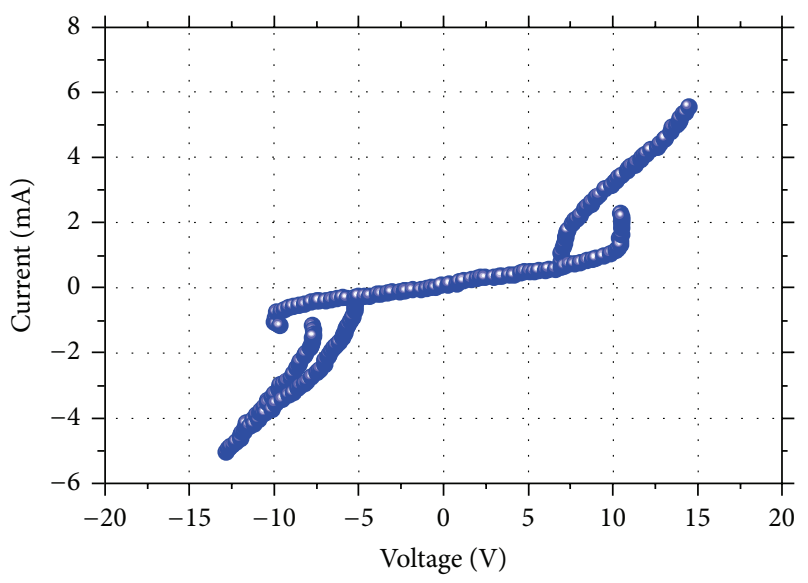

FIgURE 20: $I-V$ characteristic of Al-doped $\mathrm{TiO}_{x}$ [29].

For the insertion of $\mathrm{Al}^{3+}$ ions into the AOF, the process of doping was carried out at cathodic polarization.

For the samples doped with $\mathrm{Al}$, we encountered the effect of stabilization of the switching characteristics. The point is that usually the $I-V$ curves might spontaneously slightly change their parameters with time during operation and, moreover, after several thousand switching cycles the $S$-shaped $I-V$ curve completely degrades and switching no longer occurs. The above described behaviour is typical of the pure nondoped samples, while for the structures based on $\mathrm{AOF} \mathrm{TiO}_{2}$ :Al the behaviour is radically different. For these samples, threshold voltage is of the order of $10 \mathrm{~V}$ (Figure 20), and current-voltage characteristics demonstrate stability and long-term reproducible operation (up to $10^{10}$ cycles without a noticeable change in the threshold parameters). It can be assumed that the stabilization of the switching parameters is caused by a change in the energy-band structure of titanium dioxide as a result of doping it with aluminium $[29,106]$.

\section{Switching Mechanism}

The mechanism of threshold switching has for a long time been a matter of especial concern, and the principal issue is whether the mechanism is primarily thermal or electronic. There have also been several works treating the switching effect as a metal-insulator transition occurring in electric field (see, e.g., [30] and references therein). Application of an electric field to a Mott insulator, taking also into account the current flow (since we consider the switching effect, not a stationary field effect), will result in both the thermal generation of additional carriers due to the Joule heat and the field-induced generation. The latter is due to the autoionization caused by the Coulomb barrier lowering, a phenomenon analogous to the Poole-Frenkel (P-F) effect. When the total concentration of free carriers reaches the value of $n=n_{c}$, the transition into the metal state (i.e., switching of the structure into the ON state) occurs. In a material with the temperature-induced MIT, this switching (in a relatively low field) occurs just due to the heating of the switching channel up to $T=T_{t}$ according to the critical temperature model (3). The above mentioned value of $n_{c}$ is determined by the Mott criterion:

$$
a_{H} n_{c}^{1 / 3} \approx 0.25,
$$

where $a_{H}=\varepsilon \hbar^{2} / m^{*} e^{2}$ is the effective Bohr radius, $\varepsilon$ is the static dielectric permittivity, and $m^{*}$ is the effective mass of charge carriers.

In the paper [30], it has been reported on the switching mechanism in vanadium dioxide. This mechanism is shown to be based on the electronically induced Mott insulatorto-metal transition occurring in conditions of the nonequilibrium carrier density excess in the applied electric field. The model takes into account the dependence of the carrier density on electric field (see (6) below), as well as the dependence of the critical electric field $E_{c}$ on carrier density (7) with fitting parameter $\gamma$.

For switching in $\mathrm{VO}_{2}$, the main mechanism for the fieldinduced carrier density increase is the P-F effect:

$$
n=N_{0} \exp \left(-\frac{W-\beta \sqrt{E}}{k T}\right)
$$

where $N_{0}$ is a constant independent of the field and only slightly dependent on temperature, $W$ is the conductivity activation energy, and $\beta=\left(e^{3} / \pi \varepsilon \varepsilon_{o}\right)^{1 / 2}$ is the P-F constant. Next, the dependence of $E_{c}$ on electron density has been supposed to be

$$
E_{c}=E_{0}\left(1-\frac{n}{n_{c}}\right)^{\gamma}
$$

with $n_{c}$ being the Mott critical electron density.

While the former of the two postulates (6) has afterwards been repeatedly confirmed (see, e.g., [107-113]), the latter is rather an ambiguous assumption introduced to adjust the experimental data on the $n_{m}$ values with the theory $\left(n_{m}\right.$ is the maximum electron density in the switching channel at $E=E_{\text {th }}$, where $E_{\text {th }}$ is the switching threshold voltage [30]). Actually, (7) has been somehow vindicated from the viewpoint of the scaling hypothesis inherent in a phase transition, metal-insulator transition included [114].

We remind the reader that the dependence expressed by (7) was introduced in $[30,114]$ in order that the $n_{m}(T)$ behaviour might be comprehended. The data on the $n_{m}$ temperature dependence (Figure 21, curve 1) were obtained on the assumption of a temperature-independent carrier mobility $\mu$, and the problem of $n_{m}$ reduction at lower temperatures was a stumbling-block, since for the Mott transition the value of $n_{c}$ should be a constant. Note that, at $T>200 \mathrm{~K}$, $n_{m} \approx 3 \cdot 10^{18} \mathrm{~cm}^{-3}=n_{c}$ for $\mathrm{VO}_{2}[30]$.

Now, in the light of some recent data on the $\mu(T)$ dependence of vanadium dioxide [115-117], the problem outlined above could be revisited. It has been shown [115] that the conductivity of $\mathrm{VO}_{2}$ can be written as

$$
\sigma \sim \frac{1}{T^{3 / 2}} \exp \left(-\frac{E_{a}}{k_{B} T}+\frac{k_{B} T}{\varepsilon}\right),
$$




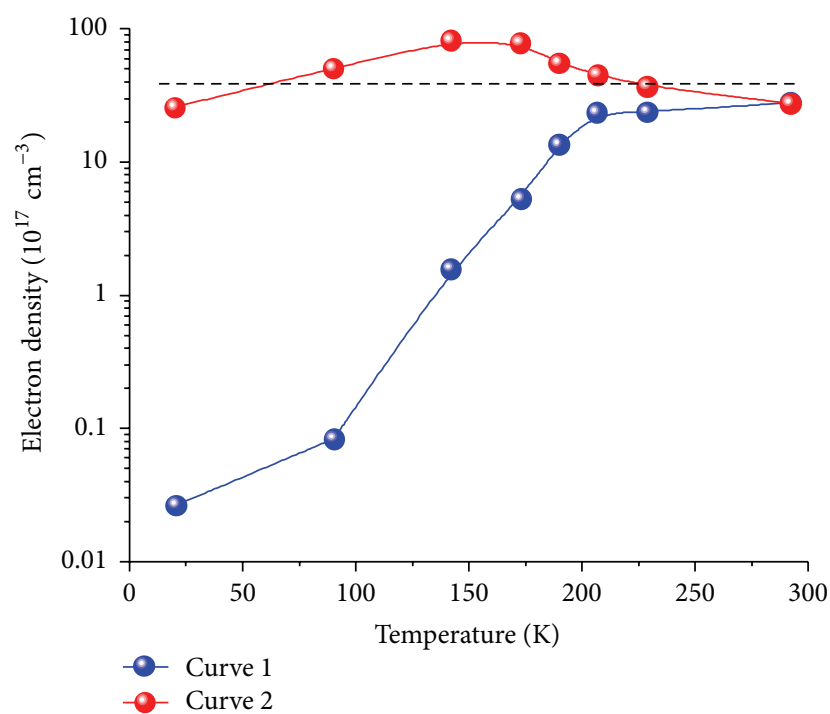

FIgURE 21: Maximum electron density in the switching channel as a function of ambient temperature; in fact, $n_{m}$ depends on the threshold electric field $E_{\text {th }}$, and the latter, in turn, depends on $T_{0}$. The calculations are made for the cases of $\mu=$ const (curve 1) and $\mu \sim$ $T_{m}{ }^{-1} \exp \left(k_{B} T_{m} / \varepsilon\right)$ (curve 2). Here $T_{m}\left(\neq T_{0}\right)$ is the temperature in the channel centre calculated on the basis of mathematical simulating [30].

where $E_{a}$ is the conductivity activation energy and $\varepsilon$ is a constant of the small polaron hopping conduction theory which takes into account the influence of thermal lattice vibrations onto the resonance integral [118]. This behaviour of $\sigma(T)$ has first been shown to be valid for $\mathrm{VO}_{2}$ single crystals [115] and then for vanadium dioxide switching channels [116] and thin films [117] as well. It is pertinent to note here that similar results have also been obtained for some other vanadium oxides exhibiting metal-insulator transitions, namely, for $\mathrm{V}_{2} \mathrm{O}_{3}$ [119], $\mathrm{V}_{4} \mathrm{O}_{7}$ [120], $\mathrm{V}_{3} \mathrm{O}_{5}$ [121], and $\mathrm{V}_{6} \mathrm{O}_{11}$ [122].

In (8), the first term in the exponent is responsible for the band conduction, whereas the second one characterizes the temperature dependence of the hopping mobility. Therefore, the data on $n_{m}(T)$ (Figure 21, curve 1) should be corrected by means of division by a factor corresponding to the mobility temperature dependence $\mu(T) \sim(1 / T) \exp \left(k_{B} T / \varepsilon\right)$; note that the preexponential factor for mobility is proportional to $T^{-1}$, unlike that for conductivity in (8) where it is proportional to $T^{-3 / 2}$ [118]. These corrected values of $n_{m}$, for $k_{B} / \varepsilon=0.35 \mathrm{~K}^{-1}$ [115], are presented by curve 2 in Figure 21 . One can see that, within an order of magnitude, these values correspond to the Mott critical concentration (dotted line in Figure 21).

In conclusion, we emphasize that the present amendment does not call into question the results presented in [30] but merely refines upon them. In particular, it does not abolish the hypothesis concerning the scaling of $E_{c}$ which has been put forward in $[30,114]$, but parameter $\gamma$ can change; for example, it can decrease down to almost zero. Thus, like in the work [30], we can state that the switching mechanism is based on the electronically induced Mott metal-insulator transition taking into consideration the dependence of the carrier density on electric field (6) and the parametrical dependence of the critical field on current density (7).

A more detailed analysis of the results would have been possible on the basis of experimental data on the vanadium dioxide mobility temperature dependence. In the recent studies $[123,124]$, the values of $\mu \sim 0.1-0.2 \mathrm{~cm}^{2} \mathrm{~V}^{-1} \mathrm{~s}^{-1}$ (at room temperature) have been obtained, which is really close to an estimate $\mu=0.5 \mathrm{~cm}^{2} \mathrm{~V}^{-1} \mathrm{~s}^{-1}$ used for calculations in [30]. However, the measurements in these works have been performed in a limited temperature range (in the vicinity of the transition), where the mobility only slightly depends on temperature $[123,124]$.

In recent literature, much attention is paid to the problem of relative contributions of thermal and electronic effects in switching, mainly in connection with the development of a "Mott-transition FET" [125]. Particularly, the author of [126] compares FETs based on strongly correlated TMOs and those on $\mathrm{Si}$. The devices on the basis of $\mathrm{SmNiO}_{3}, \mathrm{VO}_{2}$, and $\mathrm{LaAlO}_{3}$ $\mathrm{SrTiO}_{3}$ are examined. As for the vanadium dioxide, it is stated, with the reference to the work [127], that "in $\mathrm{VO}_{2}$ the transition is in large part caused by micrometer-scale heating by the applied electric field." It should be stressed however that thermal effects do take place at switching (not at the field effect) [30], and it has always been known that the voltage-induced MIT in macroscopic $\mathrm{VO}_{2}$ crystals and planar micron-size film structures occurs due to the Joule heating effect $[128,129]$. Note that a "micrometer-scale" size is surely macroscopic with respect to nanoscale dimensions. Therefore, the results of [127] do not contradict the idea of the field-controlled MIT in $\mathrm{VO}_{2}$. In summary, one can arrive at a conclusion that the switching mechanism involves a combination of both thermal and electronic effects, and their relative contributions depend on device dimensions and ambient temperature $[30,113,130]$. For example, at a relatively high temperature, close to $T_{t}$, the mechanism is merely thermal and switching is described by the critical temperature model (3). On the other hand, in some cases (nanoscale dimensions and low temperatures) switching might be completely electronically induced. This conclusion is supported by experiments on electric field-stimulated Mott transition in $\mathrm{VO}_{2}[125,131-133]$. Also, separating electric field and thermal effects in vanadium oxide nanobeams has been recently demonstrated in [134].

In addition, valuable information about the contributions of electronic and thermal effects in switching might be obtained when investigating the switching delay time [131]. For example, electrical switching dynamics of $\mathrm{VO}_{2}$ in the $\mathrm{RF}$ range has been studied in the works $[135,136]$. Particularly, two distinguishable time scales for electrical switching have been observed [135], one in accordance with Joule heating and one much faster, which may suggest contribution of the electronic Mott MIT-based effects.

Finally, we note that electronically induced switching associated with the MIT has been reported for a number of other materials (not only for $\mathrm{VO}_{2}$ ), for example, in a family of Mott insulators $\mathrm{AM}_{4} \mathrm{X}_{8}(\mathrm{~A}=\mathrm{Ga}, \mathrm{Ge} ; \mathrm{M}=\mathrm{V}, \mathrm{Nb}, \mathrm{Ta} ; \mathrm{X}=\mathrm{S}$, $\mathrm{Se})[137,138], \mathrm{SmNiO}_{3}[139], \beta-\mathrm{Pb}_{x} \mathrm{~V}_{2} \mathrm{O}_{5}$ [140], and $\mathrm{H}$-doped $\mathrm{NbO}_{2}[141]$. 


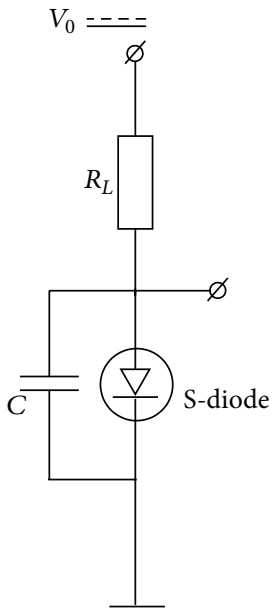

(a)

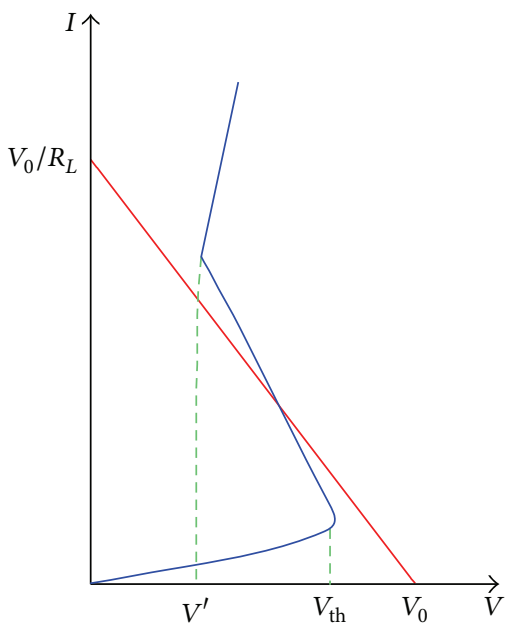

(b)

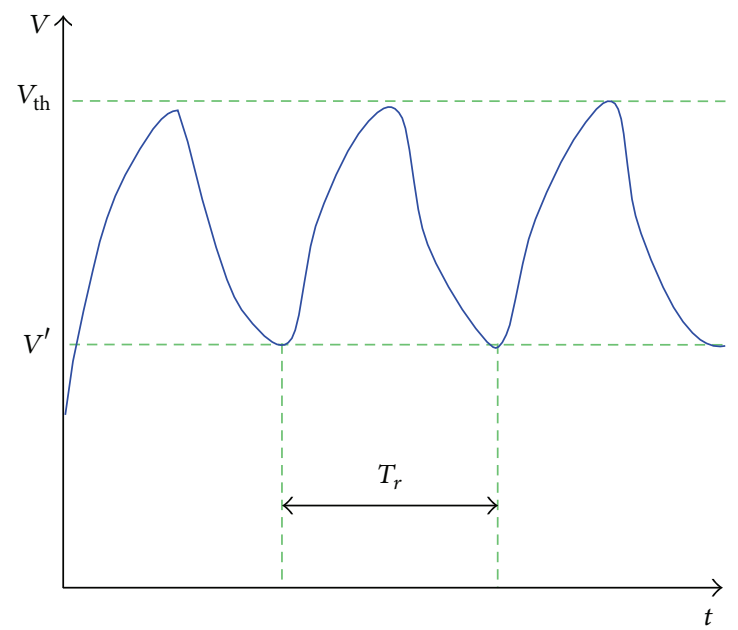

(c)

FIGURE 22: Relaxation oscillations: (a) electrical circuit, (b) schematic $I-V$ curve and load line, and (c) output signal oscillogram.

\section{Potential Applications of Switching in Transition Metal Oxides}

Devices with negative differential resistance (S- or $\mathrm{N}$-shaped $I-V$ characteristics) have potential applications in electronics as switches, memory elements, and microsensors [142]. One more recently emerging field of application is connected with neurochips and neural networks on the basis of memristors [125], including those made of TMO-based switching elements. Particularly, a $\mathrm{NbO}_{2}$-based switching device is considered in [100] in this regard.

In a scheme with an S-NDR device, at a high enough d.c. bias $\left(V_{0}\right)$, relaxation oscillations are observed if the load line intersects with the $I-V$ curve at a unique point in the negative resistance region (Figure 22) [142]. The frequency of this oscillation $\left(f_{r}=1 / T_{r}\right)$ depends on the threshold parameters and, consequently, it depends on external parameters, for example, on temperature and pressure. Sensors with the frequency output have a number of evident advantages: high sensitivity and stability, convenience of processing of a signal, capability of remote measurements, and low noise susceptibility. The $f_{r}(T)$-output temperature sensor based on $\mathrm{VO}_{2}$ has been first proposed in [143] and then developed in [144].

Electrical self-oscillations across out-of-plane threshold switches based on $\mathrm{VO}_{2}$ have also been recently studied in the article [145]. The authors of this work note that, beside integration as inverters and oscillators in highspeed circuits, a prospective potential application may be the parallel production of periodic signals in large scale integrated circuits, analogous to the action potentials in neural architectures. Note that, in the work [100], a MITbased switching devise has been proposed to be used as a quasistatic neuristor synapse model. On the other hand, dynamic oscillation-based models are also widely explored in the field of neuromorphic simulation [146-149]. Particularly, in the works $[148,149]$, the authors present the realization of coupled and scalable relaxation oscillators utilizing the MIT in metal-insulator-metal transition in $\mathrm{VO}_{2}$ and demonstrate the potential use of such a system in pattern recognition as one possible computational model based on a non-Boolean neuromorphic computing paradigm. Further simulation of computing with networks of synchronous oscillators based on MIT-devices has been recently presented in [150]. Note that thermally induced oscillations $[151,152]$, resistance noise [153], negative capacitance [154], and some other possible concomitant phenomena should apparently be taken into account when constructing neuromorphic circuits on the basis of switching devices with a MIT-induced NDR.

The development of the ideas expressed in [100] goes on the way of both analysis of the various schemes [155] and mathematical models [156] for constructing such a "memristor-based neuristor," as well as offering a variety of materials (mainly TMOs) to create a memristor. In view of the above-said, the following research area seems to be promising, namely, investigate the possibility of applying the switching effect in structures based on $\mathrm{VO}_{2}$ (and possibly other TMOs) to simulate the function of the neuristor synapse. Some of these materials and devices for adaptive oxide electronics have been recently reviewed in [157]. Additional capabilities are provided by a combination of memory and MIT-induced switching in one device on the basis of vanadium dioxide [158], see Figure 23. It should be noted that bipolar memory switching in disordered vanadium oxide has earlier been reported in the work [159].

To implement these ideas, one can use a memristive device based on niobium oxide $\mathrm{NbO}_{2}$ [100]. As was discussed above in Section 2, Mott insulators such as $\mathrm{NbO}_{2}$ have long been known to exhibit current-controlled NDR. This phenomenon is caused by a reversible metal-insulator phase transition. However, due to relatively high transition temperature (1070 K [24]), niobium dioxide consumes a significant amount of energy which evolved as heat. Therefore, it is not yet possible to arrange a plurality of available neuristors with high density on a single chip. Vanadium dioxide, on the other hand, with the MIT temperature of only $340 \mathrm{~K}$ seems to be a more attractive material for this application. Among other 


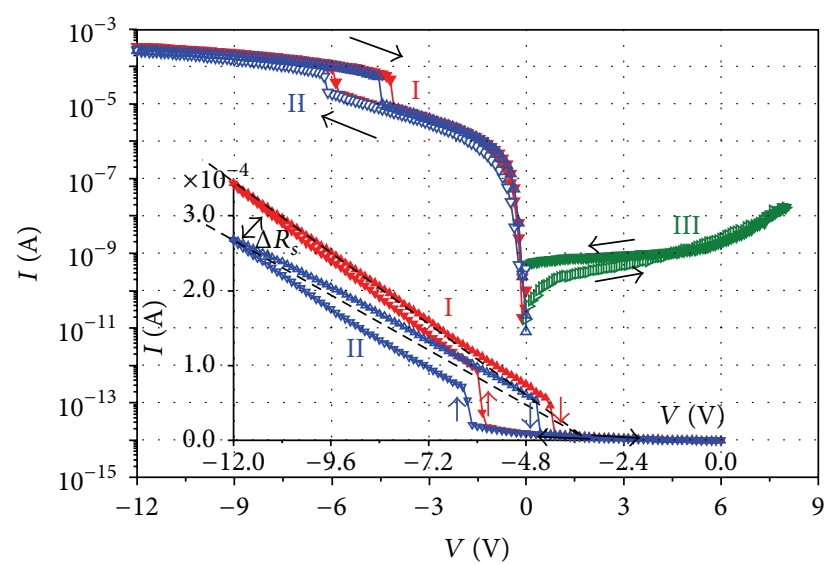

FIGURE 23: $I-V$ characteristic of $\mathrm{VO}_{2}$ on $\mathrm{p}$-Si after final electroforming, demonstrating both MIT-induced threshold (I, II) and iondiffusion-based memory (III) switching (inset, the same curve at negative polarity in linear coordinates).

materials for neuromorphic memristive circuits and adaptive systems, one can mention also, for example, $\mathrm{Li}_{x} \mathrm{CoO}_{2}$ [160], $\mathrm{MoO}_{x} / \mathrm{MoS}_{2}$, and $\mathrm{WO}_{x} / \mathrm{WS}_{2}$ heterostructures [161], and some other binary and complex TMOs [157]. In conclusion, we would like to mention also a recent work discussing general aspects of neuromorphic networks based on metaloxide memristors [162]. On the other hand, dynamical oscillator-based networks are reviewed in [163].

Other possible applications of the switching effect in $\mathrm{VO}_{2}$ include, for example, microwave switching devices [135, $136,164]$, thermally and optically biased memristive switches [165], electrolyte gated THz photonic devices [166], and thinfilm varistors [167] (see also some examples in reviews [125, $157,168,169])$. In addition, switching structures, particularly those based on $\mathrm{Nb}$ and $\mathrm{Ti}$ oxides, can be used as access elements (selective devices) in high density cross-point type ReRAMs [170, 171].

\section{Conclusion}

Nonlinear electric conduction in strongly correlated electron systems exhibiting metal-insulator phase transitions is characterized by negative differential resistivity $[2,172]$. The theoretical difficulties in the study of NDR are connected with the fact that the system is far from equilibrium owing to the dissipation caused by the finite current, and nonperturbative analysis is necessary if the NDR is associated with the threshold property such as the metal-insulator transition [172].

In this review, we have confined our attention mostly to S-type threshold switching (S-NDR) in oxides of transition metals. Electrical instabilities with voltage-controlled $(\mathrm{N}-$ type) NDR and various memory effects have also been reported by many researchers both in oxides and in other materials. However, "pure" threshold switching with an Sshaped $I(V)$ curve, aside from chalcogenide semiconductors, is characteristic of a number of transition metal oxides. This is closely linked to the electronic structure of the transition metals. A set of valence states, associated with the existence of unfilled $d$-shells in these atoms, leads to formation of several oxide phases with different properties [23, 25, 26, 173], ranging from metallic to insulating. This accounts for the ease of chemical transformations during electroforming. On the other hand, it is specifically the behaviour of $d$-electrons in the compounds of transition metals that is responsible for the unique properties of these materials, causing strong electronelectron and electron-phonon correlations, which play an important role in the mechanism of MITs.

The results presented in Sections 2 and 3 of this review indicate that current instabilities with the S- or N-NDR exhibit several common features. In particular, for each of the investigated materials there is a certain fixed temperature $T_{0}$, above which switching disappears. At $T<T_{0}$, the threshold voltage decreases as the temperature rises, tending to zero at $T \rightarrow T_{0}$. Comparison of these temperatures with the temperatures of MIT for some compounds (e.g., $T_{t}=340 \mathrm{~K}$ for $\mathrm{VO}_{2}$, etc.) shows that the switching effect is associated with the insulator-to-metal transition in an electric field. The channels, consisting of these lower oxides, are formed in initial films during preliminary electroforming.

Note that formation of Ti lower oxides $\left(\mathrm{Ti}_{n} \mathrm{O}_{2 n-1}\right.$ Magneli phases) during EF has also been reported in a number of works on memory switching in titanium oxide systems [174, 175]. The same is true for the $\mathrm{Ta}-\mathrm{O}$ system where $\mathrm{TaO}_{x}$ suboxides have been reported $[49,176]$. Moreover, $\mathrm{TaO}_{2}$ exhibiting a Peierls MIT has been recently reported to exist [177].

Since electronic devices that exhibit NDR are useful in electric circuits, the understanding of NDR is also important from the viewpoint of industrial applications [172]. As we have shown in Section 4 above, vanadium dioxide appears to be the most convenient material for various applications due to the fact that its $T_{t}$ is close to room temperature. Basically, the roster of materials exhibiting MITs with sharply discontinuous switching of electrical conductivity close to room temperature remains rather sparse [140]. Apart from $\mathrm{VO}_{2}$, useful devices in this sense might turn out to be those based on manganese and molybdenum oxides (see Section 2.3), as well as $\beta-\mathrm{Pb}_{x} \mathrm{~V}_{2} \mathrm{O}_{5}(x \approx 0.33)$ [140] and hydrogen-treated $\mathrm{NbO}_{2}$ with reduced $T_{t}$ [141].

\section{Conflict of Interests}

The authors declare that there is no conflict of interests regarding the publication of this paper.

\section{Acknowledgments}

This work was supported by the RF Ministry of Education and Science as a base part of state Program no. 2014/154 in the scientific field, Project no. 1426, and State Program no. 3.757.2014/K.

\section{References}

[1] S. R. Ovshinsky, "Reversible electrical switching phenomena in disordered structures," Physical Review Letters, vol. 21, no. 20, pp. 1450-1453, 1968. 
[2] E. Schöll, Nonequilibrium Phase Transitions in Semiconductors. Self-Organization Induced by Generation and Recombination Processes, Springer, Berlin, Germany, 1987.

[3] B. T. Kolomiets, "Vitreous semiconductors", Physica Status Solidi (B), vol. 7, no. 2, pp. 359-372, 1964.

[4] A. Pergament, G. Stefanovich, A. Velichko, V. Putrolainen, T. Kundozerova, and T. Stefanovich, "Novel hypostasis of old materials in oxide electronics: metal oxides for resistive random access memory applications," Journal of Characterization and Development of Novel Materials, vol. 4, no. 2, pp. 83-110, 2011.

[5] G. W. Burr, M. J. Breitwisch, M. Franceschini et al., "Phase change memory technology," Journal of Vacuum Science and Technology B, vol. 28, no. 2, pp. 223-262, 2010.

[6] G. Dearnaley, A. M. Stoneham, and D. V. Morgan, "Electrical phenomena in amorphous oxide films," Reports on Progress in Physics, vol. 33, no. 3, pp. 1129-1191, 1970.

[7] K. L. Chopra, "Avalanche-induced negative resistance in thin oxide films," Journal of Applied Physics, vol. 36, no. 1, pp. 184187, 1965.

[8] D. P. Oxley, "Electroforming, switching and memory effects in oxide thin films," ElectroComponent Science and Technology, vol. 3, no. 4, pp. 217-224, 1977.

[9] Y. Zhu and M. Li, "Bipolar resistive switching characteristic of epitaxial $\mathrm{NiO}$ thin film on $\mathrm{Nb}$-doped $\mathrm{SrTiO}_{3}$ substrate," Advances in Condensed Matter Physics, vol. 2012, Article ID 364376, 8 pages, 2012.

[10] M.-J. Lee, S. I. Kim, C. B. Lee et al., "Low-temperaturegrown transition metal oxide based storage materials and oxide transistors for high-density non-volatile memory," Advanced Functional Materials, vol. 19, no. 10, pp. 1587-1593, 2009.

[11] B. Lalevic, N. Fuschillo, and W. Slusark Jr., "Switching in Nb$\mathrm{Nb}_{2} \mathrm{O}_{5}$ - $\mathrm{Nb}$ devices with doped $\mathrm{Nb}_{2} \mathrm{O}_{5}$ amorphous films," IEEE Transactions on Electron Devices, vol. ED-22, no. 10, pp. 965967, 1975.

[12] G. C. Vezzoli, "Recovery curve for threshold-switching $\mathrm{NbO}_{2}$," Journal of Applied Physics, vol. 50, no. 10, pp. 6390-6395, 1979.

[13] S. H. Shin, T. Halpern, and P. M. Raccah, "High-speed highcurrent field switching of $\mathrm{NbO}_{2}$," Journal of Applied Physics, vol. 48, no. 7, article 3150, pp. 3150-3153, 1977.

[14] G. C. Vezzoli, P. J. Walsh, and M. A. Shoga, "Interpretation of recent transient on-state data in thin chalcogenide glass and $\mathrm{NbO}_{2}$ threshold switching material," Philosophical Magazine B, vol. 63, no. 3, pp. 739-755, 1991.

[15] G. Taylor and B. Lalevic, "RF relaxation oscillations in polycrystalline $\mathrm{TiO}_{2}$ thin films," Solid State Electronics, vol. 19, no. 8, pp. 669-674, 1976.

[16] B. P. Zakharchenya, V. P. Malinenko, G. B. Stefanovich, M. Y. Terman, and F. A. Chudnovskii, "Switching in MOM structures based on vanadium dioxide," Soviet Technical Physics Letters, vol. 11, pp. 108-111, 1985.

[17] R. C. Morris, J. E. Christopher, and R. V. Coleman, "Conduction phenomena in thin layers of iron oxide," Physical Review, vol. 184, no. 2, pp. 565-573, 1969.

[18] A. E. Owen, P. G. Le Comber, J. Hajto, M. J. Rose, and A. J. Snell, "Switching in amorphous devices," International Journal of Electronics, vol. 73, no. 5, pp. 897-906, 1992.

[19] A. K. Ray and C. A. Hogarth, "A critical review of the observed electrical properties of MIM devices showing VCNR," International Journal of Electronics, vol. 57, no. 1, pp. 1-78, 1984.

[20] D. V. Morgan, M. J. Howes, R. D. Pollard, and D. G. P. Waters, "Electroforming and dielectric breakdown in thin aluminium oxide films," Thin Solid Films, vol. 15, no. 1, pp. 123-131, 1973.
[21] S. Hirose, H. Niimi, K. Kageyama, A. Ando, H. Ieki, and T. Omata, "Impact of the electrical forming process on the resistance switching behaviors in lanthanum-doped strontium titanate ceramic chip devices," Japanese Journal of Applied Physics, vol. 52, no. 4, Article ID 045802, 2013.

[22] A. L. Pergament, G. B. Stefanovich, A. A. Velichko, and S. D. Khanin, "Electronic switching and metal-insulator transitions in compounds of transition metals," in Condensed Matter at the Leading Edge, pp. 1-67, Nova Science Publishers, 2006.

[23] P. A. Cox, Transition Metal Oxides. An Introduction to Their Electronic Structure and Properties, Clarendon Press, Oxford, UK, 1992.

[24] N. F. Mott, Metal-Insulator Transition, Taylor \& Francis, London, UK, 2nd edition, 1990.

[25] S. V. Kalinin and N. A. Spaldin, "Functional ion defects in transition metal oxides," Science, vol. 341, no. 6148, pp. 858-859, 2013.

[26] S.-W. Cheong, "Transition metal oxides: the exciting world of orbitals," Nature Materials, vol. 6, no. 12, pp. 927-928, 2007.

[27] A. L. Pergament, G. B. Stefanovich, and F. A. Chudnovskii, "Metal-semiconductor phase transition and switching effect in oxides of transition metals," Physics of the Solid State, vol. 36, no. 10, pp. 1590-1597, 1994.

[28] F. A. Chudnovskii, L. L. Odynets, A. L. Pergament, and G. B. Stefanovich, "Electroforming and switching in oxides of transition metals: the role of metal-insulator transition in the switching mechanism," Journal of Solid State Chemistry, vol. 122, no. 1, pp. 95-99, 1996.

[29] V. P. Malinenko, A. L. Pergament, O. V. Spirin, and V. I. Nikulshin, "Threshold and memory switching in oxides of molybdenum, niobium, tungsten, and titanium," Journal on Selected Topics in Nano Electronics and Computing, vol. 2, no. 2, pp. 45-49, 2014.

[30] A. L. Pergament, P. P. Boriskov, A. A. Velichko, and N. A. Kuldin, "Switching effect and the metal-insulator transition in electric field," Journal of Physics and Chemistry of Solids, vol. 71, no. 6, pp. 874-879, 2010.

[31] A. L. Pergament, V. P. Malinenko, O. I. Tulubaeva, and L. A. Aleshina, "Electroforming and switching effects in yttrium oxide," Physica Status Solidi A, vol. 201, no. 7, pp. 1543-1550, 2004.

[32] C. J. Dell'Oka, D. L. Pulfrey, and L. Young, "Anodic oxide films," in Physics of Thin Films, M. H. Francombe and R. W. Hoffman, Eds., vol. 6, pp. 1-79, Academic Press, New York, NY, USA, 1971.

[33] G. B. Stefanovich, A. L. Pergament, A. A. Velichko, and L. A. Stefanovich, "Anodic oxidation of vanadium and properties of vanadium oxide films," Journal of Physics Condensed Matter, vol. 16, no. 23, pp. 4013-4024, 2004.

[34] D. G. Lovering, Molten Salt Technology, Springer, New York, NY, USA, 1982.

[35] G. V. Samsonov, The Oxide Handbook, IFI/Plenum, New York, NY, USA, 1982.

[36] K. Goto, "On the mechanism of phase transitions of $\mathrm{UO}_{2.25}$ ", Solid State Communications, vol. 6, no. 9, pp. 653-655, 1968.

[37] F. A. Chudnovskii, "Metal-semiconductor phase transition in vanadium oxides and technical applications," Soviet PhysicsTechnical Physics, vol. 20, no. 8, pp. 999-1012, 1976.

[38] J. Duchene, "Direct infrared measurements of filament transient temperature during switching in vanadium oxide film devices," Journal of Solid State Chemistry, vol. 12, no. 3-4, pp. 303-306, 1975. 
[39] A. Mansingh and R. Singh, "The mechanism of electrical threshold switching in $\mathrm{VO}_{2}$ crystals," Journal of Physics C: Solid State Physics, vol. 13, no. 31, pp. 5725-5733, 1980.

[40] V. N. Andreev, N. E. Timoschenko, I. M. Chernenko, and F. A. Chudnovskii, "Mechanism of formation of switching vanadatephosphate glasses," Journal of Technical Physics, vol. 51, no. 8, pp. 1685-1689, 1981.

[41] J. K. Higgins, B. K. Temple, and J. E. Lewis, "Electrical properties of vanadate-glass threshold switches," Journal of NonCrystalline Solids, vol. 23, no. 2, pp. 187-215, 1977.

[42] J. G. Zhang and P. C. Eklund, "The switching mechanism in $\mathrm{V}_{2} \mathrm{O}_{5}$ gel films," Journal of Applied Physics, vol. 64, no. 2, pp. 729-733, 1988.

[43] J. Bullot, O. Gallias, M. Gauthier, and J. Livage, "Threshold switching in $\mathrm{V}_{2} \mathrm{O}_{5}$ layers deposited from gels," Physica Status Solidi (A), vol. 71, no. 1, pp. K1-K4, 1982.

[44] A. I. Ivon, V. R. Kolbunov, and I. M. Chernenko, "Voltagecurrent characteristics of vanadium dioxide based ceramics," Journal of the European Ceramic Society, vol. 23, no. 12, pp. 21132118, 2003.

[45] R. L. Remke, R. M. Walser, and R. W. Bené, "The effect of interfaces on electronic switching in $\mathrm{VO}_{2}$ thin films," Thin Solid Films, vol. 97, no. 2, pp. 129-143, 1982.

[46] R. Collongues, La Non-Stoechiometrie, Masson, Paris, France, 1971.

[47] P. Kofstad, Nonstoichiometry, Diffusion and Electrical Conductivity in Binary Metal Oxides, Wiley-Interscience, New York, NY, USA, 1972.

[48] D. R. Islamov, V. A. Gritsenko, C. H. Cheng, and A. Chin, "Percolation conductivity in hafnium sub-oxides," Applied Physics Letters, vol. 105, no. 26, Article ID 262903, 2014.

[49] W. Kulisch, D. Gilliland, G. Ceccone et al., "Tantalum pentoxide as a material for biosensors: deposition, properties and applications," in Nanostructured Materials for Advanced Technological Applications, J. P. Reithmaier, P. Petkov, W. Kulisch, and C. Popov, Eds., NATO Science for Peace and Security Series B: Physics and Biophysics, pp. 509-524, Springer Science+Business Media, Dordrecht, The Netherlands, 2009.

[50] K. D. Tséndin, É. A. Lebedev, and A. B. Shmel'kin, "Instabilities with $S$ - and $N$-shaped current-voltage characteristics and phase transitions in chalcogenide vitreous semiconductors and polymers," Physics of the Solid State, vol. 47, no. 3, pp. 439-445, 2005.

[51] A. Pergament and G. Stefanovich, "Insulator-to-metal transition in vanadium sesquioxide: does the Mott criterion work in this case?" Phase Transitions, vol. 85, no. 3, pp. 185-194, 2012.

[52] J. S. Brockman, L. Gao, B. Hughes et al., "Subnanosecond incubation times for electric-field-induced metallization of a correlated electron oxide," Nature Nanotechnology, vol. 9, no. 6, pp. 453-458, 2014.

[53] A. L. Pergament, V. P. Malinenko, L. A. Aleshina, E. L. Kazakova, and N. A. Kuldin, "Electrical switching in thin film structures based on molybdenum oxides," Journal of Experimental Physics, vol. 2014, Article ID 951297, 6 pages, 2014.

[54] A. L. Pergament, V. P. Malinenko, L. A. Aleshina, and V. V. Kolchigin, "Metal-insulator phase transition and electrical switching in manganese dioxide," Physics of the Solid State, vol. 54, no. 12, pp. 2486-2490, 2012.

[55] L. Mai, F. Yang, Y. Zhao et al., "Molybdenum oxide nanowires: synthesis and properties," Materials Today, vol. 14, no. 7-8, pp. 346-353, 2011.
[56] M. C. Rao, K. Ravindranadh, A. Kasturi, and M. S. Shekhawat, "Structural stoichiometry and phase transitions of $\mathrm{MoO}_{3}$ thin films for solid state microbatteries," Research Journal of Recent Sciences, vol. 2, no. 4, pp. 67-73, 2013.

[57] R. L. Smith and G. S. Rohrer, "Scanning probe microscopy of cleaved molybdates: $\alpha-\mathrm{MoO}_{3}(010), \quad \mathrm{Mo}_{18} \mathrm{O}_{52}(100)$, $\mathrm{Mo}_{8} \mathrm{O}_{23}(010)$, and $\eta-\mathrm{Mo}_{4} \mathrm{O}_{11}(100)$," Journal of Solid State Chemistry, vol. 124, no. 1, pp. 104-115, 1996.

[58] D. O. Scanlon, G. W. Watson, D. J. Payne, G. R. Atkinson, R. G. Egdell, and D. S. L. Law, "Theoretical and experimental study of the electronic structures of $\mathrm{MoO}_{3}$ and $\mathrm{MoO}_{2}$," Journal of Physical Chemistry C, vol. 114, no. 10, pp. 4636-4645, 2010.

[59] M. Arita, H. Kaji, T. Fujii, and Y. Takahashi, "Resistance switching properties of molybdenum oxide films," Thin Solid Films, vol. 520, no. 14, pp. 4762-4767, 2012.

[60] M. Hasan, "A materials approach to resistive switching memory oxides," Journal of Semiconductor Technology and Science, vol. 8, no. 1, pp. 66-79, 2008.

[61] A. I. Gavrilyuk and N. A. Sekushin, Electrochromism and Photochromism in Oxides of Tungsten and Molybdenum, Nauka, Leningrad, Russia, 1990, (Russian).

[62] H. Fujishita, M. Sato, S. M. Shapiro, and S. Hoshino, "Inelastic neutron scattering of the low-dimensional conductors $\left(\mathrm{TaSe}_{4}\right)_{2} \mathrm{I}$ and $\mathrm{Mo}_{8} \mathrm{O}_{23}$," Physica $B+C$, vol. 143, no. 1-3, pp. 201-203, 1986.

[63] T. Sato, T. Dobashi, H. Komatsu, T. Takahashi, and M. Koyano, "Electronic structure of $\eta-\mathrm{Mo}_{4} \mathrm{O}_{11}$ studied by high-resolution angle-resolved photoemission spectroscopy," Journal of Electron Spectroscopy and Related Phenomena, vol. 144-147, pp. 549-552, 2005.

[64] Y. Motome and N. Furukawa, "Orbital degeneracy and Mott transition in Mo pyrochlore oxides," Journal of Physics: Conference Series, vol. 320, Article ID 012060, 2011.

[65] A. Maeda, T. Furuyama, and S. Tanaka, "Threshold-field behavior and switching in $\mathrm{K}_{0.3} \mathrm{MoO}_{3}$," Solid State Communications, vol. 55, no. 11, pp. 951-955, 1985.

[66] Y.-C. Lin, D. O. Dumcenco, Y.-S. Huang, and K. Suenaga, "Atomic mechanism of the semiconducting-to-metallic phase transition in single-layered $\mathrm{MoS}_{2}$," Nature Nanotechnology, vol. 9, no. 5, pp. 391-396, 2014.

[67] B. Sun, W. Zhao, Y. Liu, and P. Chen, "Resistive switching effect of Ag/ $\mathrm{MoS}_{2} /$ FTO device," Functional Materials Letters, vol. 8, no. 1, Article ID 1550010, 4 pages, 2015.

[68] R. Rousseau, E. Canadell, P. Alemany, D. H. Galván, and R. Hoffmann, "Origin of the metal-to-insulator transition in $\mathrm{H}_{0.33} \mathrm{MoO}_{3}$," Inorganic Chemistry, vol. 36, no. 21, pp. 4627-4632, 1997.

[69] E. Canadell and M.-H. Whangbo, "Band electronic structure study of the structural modulation in the Magnéli phase $\mathrm{Mo}_{8} \mathrm{O}_{23}$," Inorganic Chemistry, vol. 29, no. 12, pp. 2256-2260, 1990.

[70] S. Mukherjee, S. Karmakar, H. Sakata, and B. K. Chaudhuri, "Low-temperature metallic behavior of amorphous $\mathrm{MoO}_{3}$ $\mathrm{TeO}_{2}$ thin films," Journal of Applied Physics, vol. 97, no. 12, Article ID 123707, 2005.

[71] A. Mottaghizadeh, Q. Yu, P. L. Lang, A. Zimmers, and H. Aubin, "Metal oxide resistive switching: evolution of the density of states across the metal-insulator transition," Physical Review Letters, vol. 112, no. 6, Article ID 066803, 2014.

[72] R. Xie, C. T. Bui, B. Varghese et al., "An electrically tuned solid-state thermal memory based on metal-insulator transition of single-crystalline $\mathrm{VO}_{2}$ nanobeams," Advanced Functional Materials, vol. 21, no. 9, pp. 1602-1607, 2011. 
[73] M. R. Arora and R. Kelly, "The structure and stoichiometry of anodic films on V, Nb, Ta, Mo and W," Journal of Materials Science, vol. 12, no. 8, pp. 1673-1684, 1977.

[74] C. M. Daly and R. G. Keil, "On the anodic oxidation of molybdenum," Journal of the Electrochemical Society, vol. 122, no. 3, pp. 350-353, 1975.

[75] A. L. Pergament and G. B. Stefanovich, "Phase composition of anodic oxide films on transition metals: a thermodynamic approach," Thin Solid Films, vol. 322, no. 1-2, pp. 33-36, 1998.

[76] R. Swanepoel, "Determination of the thickness and optical constants of amorphous silicon," Journal of Physics E: Scientific Instruments, vol. 16, no. 12, pp. 1214-1221, 1983.

[77] W.-H. Ryu, J.-H. Yoon, and H.-S. Kwon, "Morphological control of highly aligned manganese dioxide nanostructure formed by electrodeposition," Materials Letters, vol. 79, pp. 184-187, 2012.

[78] A. L. Gusev, T. N. Kondyrina, V. V. Kursheva et al., "Perspectives on application of flexible electrochromic panels in housing and communal services facilities and vehicles," International Scientific Journal for Alternative Energy and Ecology, vol. 10, no. 78, pp. 122-137, 2009.

[79] S. Walia, S. Balendhran, P. Yi et al., " $\mathrm{MnO}_{2}$-based thermopower wave sources with exceptionally large output voltages," Journal of Physical Chemistry C, vol. 117, no. 18, pp. 9137-9142, 2013.

[80] H. Wang, C. Peng, J. Zheng, F. Peng, and H. Yu, "Design, synthesis and the electrochemical performance of $\mathrm{MnO}_{2} / \mathrm{C} @ \mathrm{CNT}$ as supercapacitor material," Materials Research Bulletin, vol. 48, no. 9, pp. 3389-3393, 2013.

[81] M. K. Yang, J.-W. Park, T. K. Ko, and J.-K. Lee, "Resistive switching characteristics of TiN/ $\mathrm{MnO}_{2} / \mathrm{Pt}$ memory devices," Physica Status Solidi-Rapid Research Letters, vol. 4, no. 8-9, pp. 233-235, 2010.

[82] R. Ramesham, T. Daud, A. Moopenn, A. P. Thakoor, and S. K. Khanna, "Manganese oxide microswitch for electronic memory based on neural networks," Journal of Vacuum Science \& Technology B, vol. 7, no. 3, pp. 450-454, 1989.

[83] D. B. Rogers, R. D. Shannon, A. W. Sleight, and J. L. Gillson, "Crystal chemistry of metal dioxides with rutile-related structures," Inorganic Chemistry, vol. 8, no. 4, pp. 841-850, 1969.

[84] P. H. Klose, "Electrical properties of manganese dioxide and manganese sesquioxide," Journal of The Electrochemical Society, vol. 117, no. 7, pp. 854-859, 1970.

[85] E. L. Nagaev, Physics of Magnetic Semiconductors, Nauka, Moscow, Russia, 1979, (Russian).

[86] C.-C. Hu and T.-W. Tsou, "Ideal capacitive behavior of hydrous manganese oxide prepared by anodic deposition," Electrochemistry Communications, vol. 4, no. 2, pp. 105-109, 2002.

[87] X.-M. Shen and A. Clearfield, "Phase transitions and ion exchange behavior of electrolytically prepared manganese dioxide," Journal of Solid State Chemistry, vol. 64, no. 3, pp. 270-282, 1986.

[88] H. Y. Kang and C. C. Liang, "The anodic oxidation of manganese oxides in alkaline electrolytes," Journal of The Electrochemical Society, vol. 115, no. 1, pp. 6-10, 1968.

[89] Y. M. Hu, M. Y. Zhu, Y. Li, H. M. Jin, and Z. Z. Zhu, "Magnetic-field-assisted hydrothermal growth of manganese dioxide nanostructures and their phase transformation," Materials Science Forum, vol. 688, pp. 148-152, 2011.

[90] C. N. R. Rao and B. Raveau, Transition Metal Oxides: Structure, Properties and Synthesis of Ceramics Oxides, Wiley-VCH, New York, NY, USA, 1998.
[91] M. Regulski, R. Przeniosło, I. Sosnowska, and J.-U. Hoffmann, "Short and long range magnetic ordering in $\beta-\mathrm{MnO}_{2}-\mathrm{a}$ temperature study," Journal of the Physical Society of Japan, vol. 73, no. 12, pp. 3444-3447, 2004.

[92] V. P. Malinenko, L. A. Aleshina, S. V. Loginova, and N. D. Tikhonov, "Phase transition in pyrolytic manganese dioxide," in Proceedings of the 10th International Conference on Physics of Dielectrics, p. 39, Herzen State Pedagogical University of Russia, St. Petersburg, Russia, 2004.

[93] L. A. Aleshina and S. V. Loginova, "Total profiling of an Xray pattern of pyrolytic manganese dioxide," Russian Physics Journal, vol. 46, no. 5, pp. 500-503, 2003.

[94] A. West, Basic Solid State Chemistry, Wiley, New York, NY, USA, 1984.

[95] L. Sheng, D. Y. Xing, D. N. Sheng, and C. S. Ting, "Metalinsulator transition in the mixed-valence manganites," Physical Review B, vol. 56, no. 12, pp. R7053-R7056, 1997.

[96] A. E. Sovestnov, B. T. Melekh, V. V. Fedorov, and E. V. Fomin, "X-ray emission studies of evolution of the electron and spin structures of $\mathrm{Mn}$ in mixed manganites $\mathrm{Ln}_{1-x} \mathrm{Sr}_{x} \mathrm{MnO}_{3}(\mathrm{Ln}=\mathrm{La}$, Sm, and Ce)," Physics of the Solid State, vol. 54, pp. 778-781, 2012.

[97] E. S. Borovic, V. V. Eremenko, and A. S. Milner, Lectures on Magnetism, Fizmatlit, Moscow, Russia, 2005, (Russian).

[98] É. L. Nagaev, "Mott transitions in heavily doped magnetic semiconductors," Physics of the Solid State, vol. 40, no. 3, pp. 396-400, 1998.

[99] I. V. Borisenko, M. A. Karpov, and G. A. Ovsyannikov, "Metalinsulator transition in epitaxial films of $\mathrm{LaMnO}_{3}$ manganites grown by magnetron sputtering," Technical Physics Letters, vol. 39, no. 12, pp. 1027-1030, 2013.

[100] M. D. Pickett, G. Medeiros-Ribeiro, and R. S. Williams, "A scalable neuristor built with Mott memristors," Nature Materials, vol. 12, no. 2, pp. 114-117, 2013.

[101] S. M. Sze and K. K. Ng, Physics of Semiconductor Devices, Wiley, 2006.

[102] F. A. Chudnovskii, A. L. Pergament, D. A. Schaefer, and G. B. Stefanovich, "Effect of laser irradiation on the properties of transition metal oxides," Journal of Solid State Chemistry, vol. 118, no. 2, pp. 417-418, 1995.

[103] F. A. Chudnovskii, D. O. Kikalov, A. L. Pergament, and G. B. Stefanovich, "Electrical transport properties and switching in vanadium anodic oxides: effect of laser irradiation," Physica Status Solidi A, vol. 172, no. 2, pp. 391-395, 1999.

[104] A. L. Pergament, A. A. Velichko, O. Y. Berezina, E. L. Kazakova, N. A. Kuldin, and D. V. Artyukhin, "Influence of doping on the properties of vanadium oxide gel films," Journal of Physics Condensed Matter, vol. 20, no. 42, Article ID 422204, 2008.

[105] O. Y. Berezina, A. A. Velichko, L. A. Lugovskaya, A. L. Pergament, and G. B. Stefanovich, "Metal-semiconductor transition in nonstoichiometric vanadium dioxide films," Inorganic Materials, vol. 43, no. 5, pp. 505-511, 2007.

[106] X. Wang, Y. Song, L. L. Tao et al., "Origin of ferromagnetism in aluminum-doped $\mathrm{TiO}_{2}$ thin films: theory and experiments," Applied Physics Letters, vol. 105, no. 26, Article ID 262402, 2014.

[107] Z. Yang, S. Hart, C. Ko, A. Yacoby, and S. Ramanathan, "Studies on electric triggering of the metal-insulator transition in $\mathrm{VO}_{2}$ thin films between $77 \mathrm{~K}$ and $300 \mathrm{~K}$," Journal of Applied Physics, vol. 110, no. 3, Article ID 033725, 2011.

[108] A. Axelevitch, B. Gorenstein, and G. Golan, "Investigation of the electrical transport mechanism in $\mathrm{VO}_{\mathrm{x}}$ thin films," Microelectronics Reliability, vol. 51, no. 12, pp. 2119-2123, 2011. 
[109] G. Beydaghyan, V. Basque, and P. V. Ashrit, "High contrast thermochromic switching in vanadium dioxide $\left(\mathrm{VO}_{2}\right)$ thin films deposited on indium tin oxide substrates," Thin Solid Films, vol. 522, pp. 204-207, 2012.

[110] M. Liu, H. Y. Hwang, H. Tao et al., “Terahertz-field-induced insulator-to-metal transition in vanadium dioxide metamaterial," Nature, vol. 487, no. 7407, pp. 345-348, 2012.

[111] D.-H. Qiu, Q.-Y. Wen, Q.-H. Yang, Z. Chen, Y.-L. Jing, and H.-W. Zhang, "Electrically-driven metal-insulator transition of vanadium dioxide thin films in a metal-oxide-insulator-metal device structure," Materials Science in Semiconductor Processing, vol. 27, no. 1, pp. 140-144, 2014.

[112] G. Golan, A. Axelevitch, B. Sigalov, and B. Gorenstein, "Investigation of phase transition mechanism in vanadium oxide thin films," Journal of Optoelectronics and Advanced Materials, vol. 6, no. 1, pp. 189-195, 2004.

[113] S. Rathi, J.-H. Park, I.-Y. Lee, J. M. Baik, K. S. Yi, and G.H. Kim, "Unravelling the switching mechanisms in electric field induced insulator-metal transitions in $\mathrm{VO}_{2}$ nanobeams," Journal of Physics D: Applied Physics, vol. 47, no. 29, Article ID 295101, 2014.

[114] P. P. Boriskov, A. L. Pergament, A. A. Velichko, G. B. Stefanovich, and N. A. Kuldin, "Metal-insulator transition in electric field: a viewpoint from the switching effect," http://arxiv.org/abs/cond-mat/0603132.

[115] V. N. Andreev and V. A. Klimov, "Electrical conductivity of the semiconducting phase in vanadium dioxide single crystals," Physics of the Solid State, vol. 49, no. 12, pp. 2251-2255, 2007.

[116] A. Pergament, P. Boriskov, N. Kuldin, and A. Velichko, "Electrical conductivity of vanadium dioxide switching channel," Physica Status Solidi B, vol. 247, no. 9, pp. 2213-2217, 2010.

[117] A. Pergament, G. Stefanovich, O. Berezina, and D. Kirienko, "Electrical conductivity of tungsten doped vanadium dioxide obtained by the sol-gel technique," Thin Solid Films, vol. 531, pp. 572-576, 2013.

[118] V. V. Bryksin, "Small-polaron theory with allowance for the influence of lattice vibrations on the resonance integral," Journal of Experimental and Theoretical Physics, vol. 73, no. 5, pp. 861866, 1991.

[119] V. N. Andreev, F. A. Chudnovskiy, J. M. Honig, and P. A. Metcalf, "Electrical conductivity in the antiferromagnetic insulating phase of $\mathrm{V}_{2} \mathrm{O}_{3}$," Physical Review B, vol. 70, no. 23, Article ID 235124, 2004.

[120] V. N. Andreev and V. A. Klimov, "Specific features of electrical conductivity of $\mathrm{V}_{3} \mathrm{O}_{5}$ single crystals," Physics of the Solid State, vol. 53, no. 12, pp. 2424-2430, 2011.

[121] V. N. Andreev and V. A. Klimov, "Specific features of the electrical conductivity of $\mathrm{V}_{4} \mathrm{O}_{7}$ single crystals," Physics of the Solid State, vol. 51, no. 11, pp. 2235-2240, 2009.

[122] V. N. Andreev and V. A. Klimov, "Specific features of the electrical conductivity of $\mathrm{V}_{6} \mathrm{O}_{11}$," Physics of the Solid State, vol. 55, no. 9, pp. 1829-1834, 2013.

[123] D. Ruzmetov, D. Heiman, B. B. Claflin, V. Narayanamurti, and S. Ramanathan, "Hall carrier density and magnetoresistance measurements in thin-film vanadium dioxide across the metalinsulator transition," Physical Review B, vol. 79, no. 15, Article ID 153107, 2009.

[124] D. Fu, K. Liu, T. Tao et al., "Comprehensive study of the metalinsulator transition in pulsed laser deposited epitaxial $\mathrm{VO}_{2}$ thin films," Journal of Applied Physics, vol. 113, no. 4, Article ID 043707, 2013.
[125] A. Pergament, G. Stefanovich, and A. Velichko, "Oxide electronics and vanadium dioxide perspective: a review," Journal on Selected Topics in Nano Electronics and Computing, vol. 1, no. 1, pp. 24-43, 2013.

[126] E. S. Reich, "Metal oxide chips show promise," Nature, vol. 494, no. 7439, p. 17, 2013.

[127] A. Zimmers, L. Aigouy, M. Mortier et al., "Role of thermal heating on the voltage induced insulator-metal transition in $\mathrm{VO}_{2}$," Physical Review Letters, vol. 110, no. 5, Article ID 056601, 2013.

[128] B. S. Mun, J. Yoon, S.-K. Mo et al., "Role of joule heating effect and bulk-surface phases in voltage-driven metal-insulator transition in $\mathrm{VO}_{2}$ crystal," Applied Physics Letters, vol. 103, no. 6, Article ID 061902, 2013.

[129] S. Kumar, M. D. Pickett, J. P. Strachan, G. Gibson, Y. Nishi, and R. S. Williams, "Local temperature redistribution and structural transition during joule-heating-driven conductance switching in $\mathrm{VO}_{2}, "$ Advanced Materials, vol. 25, no. 42, pp. 6128-6132, 2013.

[130] J. Yoon, G. Lee, C. Park, B. S. Mun, and H. Ju, "Investigation of length-dependent characteristics of the voltage-induced metal insulator transition in $\mathrm{VO}_{2}$ film devices," Applied Physics Letters, vol. 105, no. 8, Article ID 083503, 2014.

[131] G. Stefanovich, A. Pergament, and D. Stefanovich, "Electrical switching and Mott transition in $\mathrm{VO}_{2}$," Journal of Physics: Condensed Matter, vol. 12, no. 41, pp. 8837-8845, 2000.

[132] M. Nakano, K. Shibuya, D. Okuyama et al., "Collective bulk carrier delocalization driven by electrostatic surface charge accumulation," Nature, vol. 487, no. 7408, pp. 459-462, 2012.

[133] T. Nan, M. Liu, W. Ren, Z.-G. Ye, and N. X. Sun, "Voltage control of metal-insulator transition and non-volatile ferroelastic switching of resistance in VOx/PMN-PT heterostructures," Scientific Reports, vol. 4, article 5931, 7 pages, 2014.

[134] A. A. Stabile, S. K. Singh, T. Wu, L. Whittaker, S. Banerjee, and G. Sambandamurthy, "Separating electric field and thermal effects across the metal-insulator transition in vanadium oxide nanobeams," Applied Physics Letters, vol. 107, no. 1, Article ID 013503, 2015.

[135] S. D. Ha, Y. Zhou, C. J. Fisher, S. Ramanathan, and J. P. Treadway, "Electrical switching dynamics and broadband microwave characteristics of $\mathrm{VO}_{2}$ radio frequency devices," Journal of Applied Physics, vol. 113, no. 18, Article ID 184501, 2013.

[136] F. Dumas-Bouchiat, C. Champeaux, A. Catherinot, A. Crunteanu, and P. Blondy, "Rf-microwave switches based on reversible semiconductor-metal transition of $\mathrm{VO}_{2}$ thin films synthesized by pulsed-laser deposition," Applied Physics Letters, vol. 91, no. 22, Article ID 223505, 2007.

[137] P. Stoliar, M. Rozenberg, E. Janod, B. Corraze, J. Tranchant, and L. Cario, "Nonthermal and purely electronic resistive switching in a Mott memory," Physical Review B, vol. 90, no. 4, Article ID 045146, 2014.

[138] P. Stoliar, L. Cario, E. Janod et al., "Universal electric-fielddriven resistive transition in narrow-gap Mott insulators," Advanced Materials, vol. 25, no. 23, pp. 3222-3226, 2013.

[139] N. Shukla, T. Joshi, S. Dasgupta, P. Borisov, D. Lederman, and S. Datta, "Electrically induced insulator to metal transition in epitaxial $\mathrm{SmNiO}_{3}$ thin films," Applied Physics Letters, vol. 105, no. 1, Article ID 012108, 2014.

[140] P. M. Marley, A. A. Stabile, C. P. Kwan et al., "Charge disproportionation and voltage-induced metal-insulator transitions evidenced in $\beta-\mathrm{Pb}_{x} \mathrm{~V}_{2} \mathrm{O}_{5}$ nanowires," Advanced Functional Materials, vol. 23, no. 2, pp. 153-160, 2013. 
[141] M. Kang, S. Yu, and J. Son, "Voltage-induced insulator-to-metal transition of hydrogen-treated $\mathrm{NbO}_{2}$ thin films," Journal of Physics D: Applied Physics, vol. 48, no. 9, Article ID 095301, 2015.

[142] A. L. Pergament, E. L. Kazakova, and G. B. Stefanovich, "Optical and electrical properties of vanadium pentoxide xerogel films: modification in electric field and the role of ion transport," Journal of Physics D: Applied Physics, vol. 35, no. 17, pp. 21872197, 2002.

[143] A. L. Pergament, G. B. Stefanovich, and F. A. Chudnovskii, "Semiconductor-metal phase transition and switching in vanadium dioxide in a high electric field," Technical Physics Letters, vol. 19, no. 10, pp. 663-665, 1993.

[144] B.-J. Kim, G. Seo, Y. W. Lee, S. Choi, and H.-T. Kim, "Linear characteristics of a metalinsulator transition voltage and oscillation frequency in $\mathrm{VO}_{2}$ devices," IEEE Electron Device Letters, vol. 31, no. 11, pp. 1314-1316, 2010.

[145] A. Beaumont, J. Leroy, J.-C. Orlianges, and A. Crunteanu, "Current-induced electrical self-oscillations across out-ofplane threshold switches based on $\mathrm{VO}_{2}$ layers integrated in crossbars geometry," Journal of Applied Physics, vol. 115, Article ID 154502, 2014.

[146] A. Belatreche, L. Maguire, M. McGinnity, L. McDaid, and A. Ghani, "Computing with biologically inspired neural oscillators: application to colour image segmentation," Advances in Artificial Intelligence, vol. 2010, Article ID 405073, 21 pages, 2010.

[147] P. Strumillo and M. Strzelecki, "Application of coupled neural oscillators for image texture segmentation and modeling of biological rhythms," International Journal of Applied Mathematics and Computer Science, vol. 16, no. 4, pp. 513-523, 2006.

[148] S. Datta, N. Shukla, M. Cotter, A. Parihar, and A. Raychowdhury, "Neuro inspired computing with coupled relaxation oscillators," in Proceedings of the 51st Annual Design Automation Conference (DAC '14), pp. 1-6, ACM, San Francisco, Calif, USA, June 2014.

[149] A. Parihar, N. Shukla, S. Datta, and A. Raychowdhury, "Exploiting synchronization properties of correlated electron devices in a non-boolean computing fabric for template matching," IEEE Journal on Emerging and Selected Topics in Circuits and Systems, vol. 4, no. 4, pp. 450-459, 2014.

[150] A. Parihar, N. Shukla, S. Datta, and A. Raychowdhury, "Synchronization of pairwise-coupled, identical, relaxation oscillators based on metal-insulator phase transition devices: a model study," Journal of Applied Physics, vol. 117, no. 5, Article ID 054902, 2015.

[151] Y. Wang, J. Chai, S. Wang et al., "Electrical oscillation in $\mathrm{Pt} / \mathrm{VO}_{2}$ bilayer strips," Journal of Applied Physics, vol. 117, no. 6, Article ID 064502, 2015.

[152] S. A. Dyakov, J. Dai, M. Yan, and M. Qiu, "Thermal selfoscillations in radiative heat exchange," Applied Physics Letters, vol. 106, no. 6, Article ID 064103, 2015.

[153] Z. Topalian, S.-Y. Li, G. A. Niklasson, C. G. Granqvist, and L. B. Kish, "Resistance noise at the metal-insulator transition in thermochromic $\mathrm{VO}_{2}$ films," Journal of Applied Physics, vol. 117, no. 2, Article ID 025303, 2015.

[154] X. He, J. Xu, X. Xu et al., "Negative capacitance switching via $\mathrm{VO}_{2}$ band gap engineering driven by electric field," Applied Physics Letters, vol. 106, no. 9, Article ID 093106, 2015.

[155] Y. Liu, T. P. Chen, Z. Liu et al., "Self-learning ability realized with a resistive switching device based on a Ni-rich nickel oxide thin film," Applied Physics A, vol. 105, no. 4, pp. 855-860, 2011.
[156] S. Wen, Z. Zeng, and T. Huang, "Exponential stability analysis of memristor-based recurrent neural networks with time-varying delays," Neurocomputing, vol. 97, pp. 233-240, 2012.

[157] S. D. Ha and S. Ramanathan, "Adaptive oxide electronics: a review," Journal of Applied Physics, vol. 110, no. 7, Article ID 071101, 2011.

[158] A. Velichko, A. Pergament, V. Putrolaynen, O. Berezina, and G. Stefanovich, "Effect of memory electrical switching in metal/vanadium oxide/silicon structures with $\mathrm{VO}_{2}$ films obtained by the sol-gel method," Materials Science in Semiconductor Processing, vol. 29, pp. 315-320, 2015.

[159] F. J. Wong, T. S. Sriram, B. R. Smith, and S. Ramanathan, "Bipolar resistive switching in room temperature grown disordered vanadium oxide thin-film devices," Solid-State Electronics, vol. 87, pp. 21-26, 2013.

[160] V. H. Mai, A. Moradpour, P. A. Senzier et al., "Memristive and neuromorphic behavior in a $\mathrm{Li}_{x} \mathrm{CoO}_{2}$ nanobattery," Scientific Reports, vol. 5, article 7761, 2015.

[161] A. A. Bessonov, M. N. Kirikova, D. I. Petukhov, M. Allen, T. Ryhänen, and M. J. A. Bailey, "Layered memristive and memcapacitive switches for printable electronics," Nature Materials, vol. 14, pp. 199-204, 2015.

[162] M. Prezioso, F. Merrikh-Bayat, B. D. Hoskins, G. C. Adam, K. K. Likharev, and D. B. Strukov, "Training and operation of an integrated neuromorphic network based on metal-oxide memristors," Nature, vol. 521, no. 7550, pp. 61-64, 2015.

[163] D. E. Nikonov, G. Csaba, W. Porod et al., "Coupled-oscillator associative memory arrayoperation," http://arxiv.org/abs/1304 .6125 .

[164] S. D. Ha, Y. Zhou, A. E. Duwel, D. W. White, and S. Ramanathan, "quick switch: strongly correlated electronic phase transition systems for cutting-edge microwave devices," IEEE Microwave Magazine, vol. 15, no. 6, pp. 32-44, 2014.

[165] G. Seo, B.-J. Kim, H.-T. Kim, and Y. W. Lee, “Thermally- or optically-biased memristive switching in two-terminal $\mathrm{VO}_{2}$ devices," Current Applied Physics, vol. 14, no. 9, pp. 1251-1256, 2014.

[166] M. D. Goldflam, M. K. Liu, B. C. Chapler et al., "Voltage switching of a $\mathrm{VO}_{2}$ memory metasurface using ionic gel," Applied Physics Letters, vol. 105, no. 4, Article ID 041117, 2014.

[167] B.-J. Kim, Y. W. Lee, S. Choi, S. J. Yun, and H.-T. Kim, " $\mathrm{VO}_{2}$ thinfilm varistor based on metal-insulator transition," IEEE Electron Device Letters, vol. 31, no. 1, pp. 14-16, 2010.

[168] Z. Yang, C. Ko, and S. Ramanathan, "Oxide electronics utilizing ultrafast metal-insulator transitions," Annual Review of Materials Research, vol. 41, pp. 337-367, 2011.

[169] Y. Zhou and S. Ramanathan, "Correlated electron materials and field effect transistors for logic: a review," Critical Reviews in Solid State and Materials Sciences, vol. 38, no. 4, pp. 286-317, 2013.

[170] S. Kim, J. Park, J. Woo et al., "Threshold-switching characteristics of a nanothin- $\mathrm{NbO}_{2}$-layer-based $\mathrm{Pt} / \mathrm{NbO}_{2} / \mathrm{Pt}$ stack for use in cross-point-type resistive memories," Microelectronic Engineering, vol. 107, pp. 33-36, 2013.

[171] D. Lee, J. Park, J. Park et al., "Structurally engineered stackable and scalable 3D titanium-oxide switching devices for highdensity nanoscale memory," Advanced Materials, vol. 27, no. 1, pp. 59-64, 2014.

[172] S. Nakamura, "Negative differential resistivity from holography," Progress of Theoretical Physics, vol. 124, no. 6, pp. 1105-1114, 2010. 
[173] E. Dagotto, "Complexity in strongly correlated electronic systems," Science, vol. 309, no. 5732, pp. 257-262, 2005.

[174] D.-H. Kwon, K. M. Kim, J. H. Jang et al., "Atomic structure of conducting nanofilaments in $\mathrm{TiO}_{2}$ resistive switching memory," Nature Nanotechnology, vol. 5, no. 2, pp. 148-153, 2010.

[175] J. J. Yang, J. P. Strachan, F. Miao et al., "Metal/TiO2 interfaces for memristive switches," Applied Physics A, vol. 102, no. 4, pp. 785-789, 2011.

[176] K. M. Kim, S. R. Lee, S. Kim, M. Chang, and C. S. Hwang, "Self-limited switching in $\mathrm{Ta}_{2} \mathrm{O}_{5} / \mathrm{TaO}_{x}$ memristors exhibiting uniform multilevel changes in resistance," Advanced Functional Materials, vol. 10, pp. 1527-1534, 2015.

[177] L. Zhu, J. Zhou, Z. Guo, and Z. Sun, "Realization of a reversible switching in $\mathrm{TaO}_{2}$ polymorphs via Peierls distortion for resistance random access memory," Applied Physics Letters, vol. 106, Article ID 091903, 2015. 

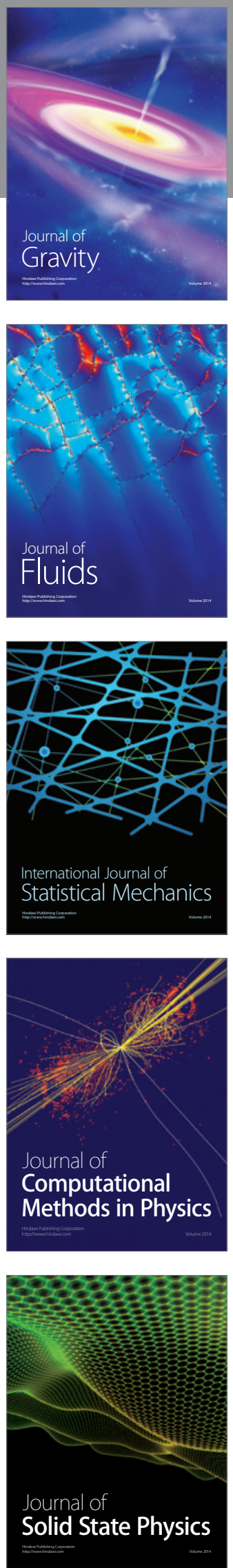

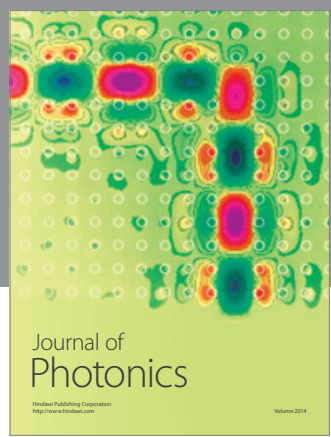

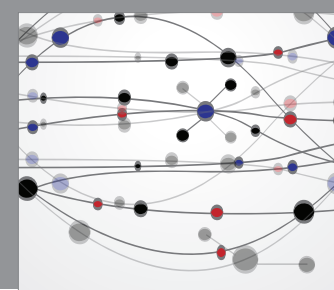

The Scientific World Journal

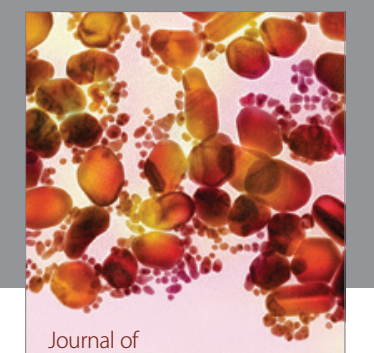

Soft Matter
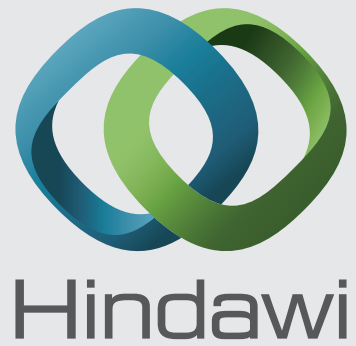

Submit your manuscripts at

http://www.hindawi.com
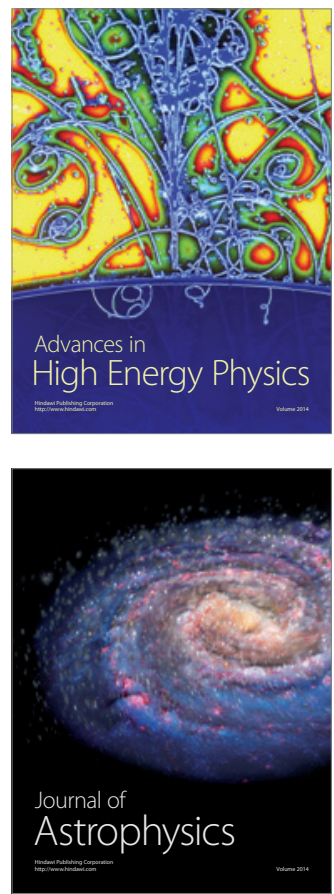
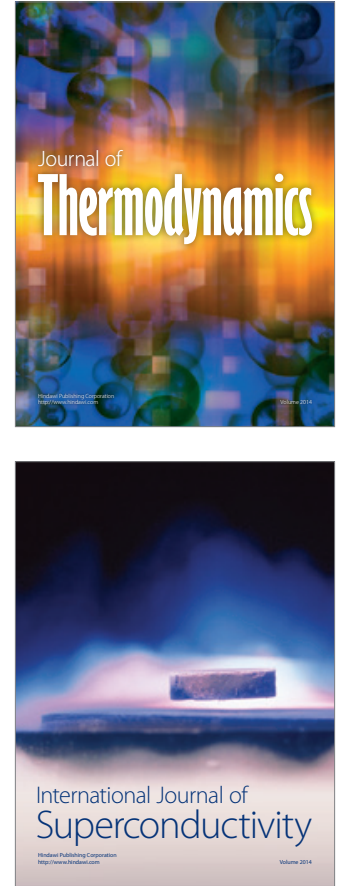
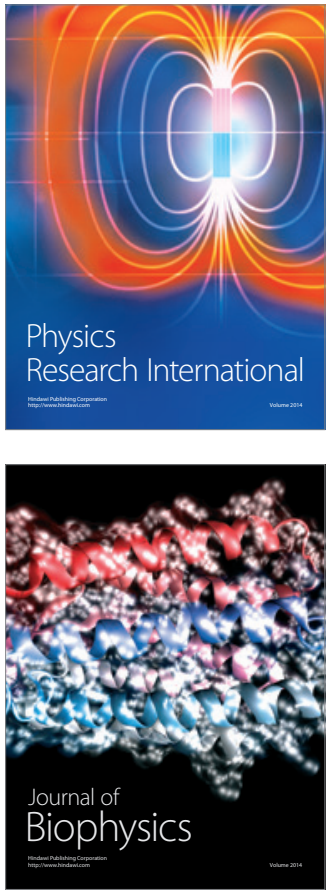
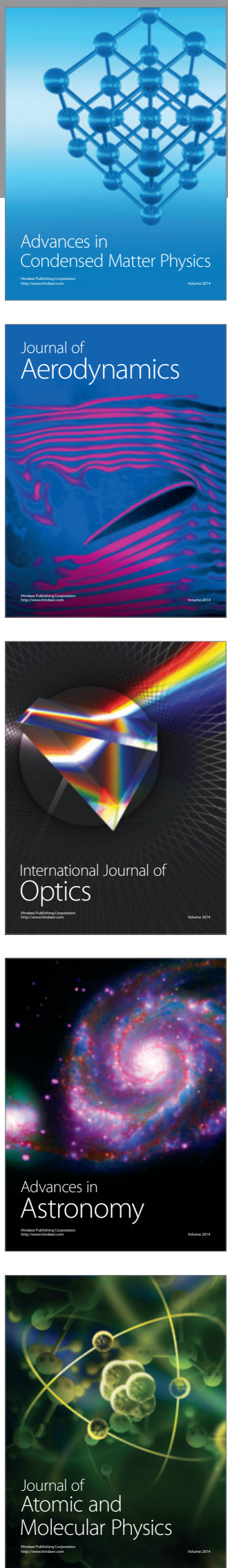